\title{
CALR frameshift mutations in MPN patient-derived iPS cells accelerate maturation of megakaryocytes
}

Kathrin Olschok ${ }^{1,2, \#, ~ L i j u a n ~ H a n ~ 1,2,3, \#, ~ M a r c e l o ~ A . ~ S . ~ d e ~ T o l e d o, ~}{ }^{1,2}$, Janik Böhnke ${ }^{4,5}$, Martin Graßhoff $^{5}$, Ivan G. Costa ${ }^{5}$, Alexandre Theocharides ${ }^{6}$, Angela Maurer ${ }^{1,2}$, Herdit M. Schüler ${ }^{7}$, Eva Miriam Buhl ${ }^{8}$, Kristina Pannen ${ }^{1,2}$, Julian Baumeister ${ }^{1,2}$, Milena Kalmer ${ }^{1,2}$, Siddharth Gupta $^{1,2}$, Peter Boor ${ }^{8}$, Deniz Gezer ${ }^{1,2}$, Tim H. Brümmendorf ${ }^{1,2}$, Martin Zenke ${ }^{4,5}$, Nicolas Chatain ${ }^{1,2,{ }^{*}} \&$ Steffen Koschmieder ${ }^{1,2, *}$

${ }^{1}$ Department of Hematology, Oncology, Hemostaseology, and Stem Cell Transplantation, Faculty of Medicine, RWTH Aachen University, Aachen, Germany

${ }^{2}$ Center for Integrated Oncology Aachen Bonn Cologne Düsseldorf (CIO ABCD)

${ }^{3}$ Department of Oncology, The First Affiliated Hospital of Zhengzhou University, Zhengzhou, China

${ }^{4}$ Institute for Biomedical Engineering, Department of Cell Biology, Faculty of Medicine, RWTH Aachen University, Aachen, Germany

${ }^{5}$ Institute for Computational Genomics Joint Research Center for Computational Biomedicine, Faculty of Medicine, RWTH Aachen University, Aachen, Germany

${ }^{6}$ Division of Hematology, University Hospital Zurich and University of Zurich, Zurich,

Switzerland

${ }^{7}$ Institute for Human Genetics, Faculty of Medicine, RWTH Aachen University, Aachen, Germany

${ }^{8}$ Institute for Pathology, Electron Microscopy Facility, Faculty of Medicine, RWTH Aachen University, Aachen, Germany

\#Shared first authorship

*Shared senior authorship

Correspondence: Prof. Dr. med. Steffen Koschmieder, Department of Hematology, Oncology, Hemostaseology, and Stem Cell Transplantation, Faculty of Medicine, RWTH Aachen University, Pauwelsstr. 30, D-52074 Aachen, Germany, Phone: +49-241-8036102; Email: skoschmieder@ukaachen.de 


\begin{abstract}
Calreticulin (CALR) mutations are driver mutations in myeloproliferative neoplasms (MPNs), leading to activation of the thrombopoietin receptor, and causing abnormal megakaryopoiesis. Here, we generated patient-derived CALRins5- or CALRdel52-positive induced pluripotent stem (iPS) cells to establish a MPN disease model for molecular and mechanistic studies. We demonstrated myeloperoxidase deficiency in CD15+ granulocytic cells derived from homozygous CALR-mutant iPS cells, rescued by repairing the mutation using CRISPR/Cas9. iPS cell-derived megakaryocytes showed characteristics of primary megakaryocytes such as formation of demarcation membrane system and cytoplasmic pro-platelets protrusions. Importantly, CALR mutations led to enhanced megakaryopoiesis and accelerated megakaryocytic development in a thrombopoietin-independent manner. Mechanistically, our study identified differentially regulated pathways in mutated vs. unmutated megakaryocytes, such as hypoxia signaling, which represents a potential target for therapeutic intervention. Altogether, we demonstrate key aspects of mutated CALR-driven pathogenesis, dependent on its zygosity and found known and novel therapeutic targets, making our model a valuable tool for clinical drug screening in MPNs.
\end{abstract}

Key words: Myeloproliferative neoplasm (MPN), induced pluripotent stem (iPS) cells, calreticulin mutation, ins5, del52, megakaryocytes, thrombopoietin, RNA sequencing, transcriptional profiling, proplatelets, essential thrombocythemia $(\mathrm{ET})$, primary myelofibrosis (PMF) 


\section{Introduction}

Myeloproliferative neoplasms (MPNs) are a group of clonal hematopoietic disorders including polycythemia vera (PV), essential thrombocythemia (ET), and primary myelofibrosis (PMF), characterized by their excessive increase of granulomonocytic cells, erythroid cells, and/or platelets as well as different degrees of splenomegaly and bone marrow (BM) fibrosis (Campo et al., 2011). Somatic calreticulin (CALR) mutations were discovered in patients with ET and PMF and have been shown to be mutually exclusive with Janus kinase 2 (JAK2) and thrombopoietin (TPO) receptor (MPL) mutations (Klampfl et al., 2013; Nangalia et al., 2013). The most common types of CALR mutations are 52 bp deletions (del52; type 1) and 5 bp insertions (ins5; type 2), all leading to $\mathrm{a}+1$ frameshift and a common novel $\mathrm{C}$ terminus of the CALR mutant protein (Klampfl et al., 2013; Nangalia et al., 2013). Although most CALR mutations are heterozygous, homozygous mutations have been observed and shown to be derived from a copy-neutral loss of heterozygosity of chromosome 19p (Klampfl et al., 2013; Stengel et al., 2019; Theocharides et al., 2016).

The oncogenic functions of CALR mutant proteins rely on its binding to the TPO receptor. This leads to the constitutive activation of JAK2 downstream targets such as STAT5, ERK1/2 and AKT, thus causing cellular transformation and abnormal megakaryopoiesis, a hallmark of CALR mutation positive MPNs (Araki et al., 2016; Chachoua et al., 2016; Han et al., 2016; Kollmann et al., 2017; Marty et al., 2016). Megakaryocytes (MKs) are platelet releasing cells, which are known to undergo different stages of maturation (George, 2000; Ru et al., 2015), but the impact of CALR mutations on megakaryocytic differentiation and function is still incompletely understood.

It has been reported that patients carrying homozygous CALRins 5 exhibit myeloperoxidase (MPO) deficiency as a result of a posttranscriptional mechanism, most likely due to defective CALR chaperone function (Nauseef et al., 1995; Theocharides et al., 2016). Additionally, a $C A L R$ knock-in mouse model demonstrated that homozygous CALRdel52 expression resulted in severe thrombocytosis and a myelofibrosis (MF) phenotype (Li et al., 2018). However, the pathogenetic impact of $C A L R$ mutant zygosity on hematopoietic and megakaryocytic differentiation has not yet been fully addressed.

Several $C A L R$ mutant disease models have been used in order to uncover its molecular and cellular mechanisms in the pathogenesis of MPN. CALR mutant overexpressing cell line models are widely used as the cells can be grown indefinitely in form of clonal populations (Araki et al., 2016; Chachoua et al., 2016; Elf et al., 2016; Han et al., 2016). However, these cell lines are derived from undifferentiated blast populations and, thus, their capacity to differentiate into hematopoietic lineages is limited. MARIMO cells, the only human cell line to date harboring mutated CALR (Kollmann et al., 2015), have been reported to carry an 
additional NRAS Q61K mutation which is responsible for transformation, while mutant $C A L R$ was not essential for these cells (Han et al., 2018). Therefore, the validity of this cell line as a model for $C A L R$ mutation analysis is questionable. Mouse models are invaluable tools for modelling human disease in vivo. Transgenic (Benlabiod et al., 2020; Li et al., 2018; Shide et al., 2019) or retrovirally transduced (Elf et al., 2016; Marty et al., 2016) mutant CALR expressing mice have been generated showing MPN-like phenotypes. However, the physiological and genetic differences between species might hamper the translation of human disease phenotypes in the mouse. For these reasons, CALR mutant MPN patient samples are desirable for analyzing biological process underlining these diseases. However, the clonal heterogeneity of hematopoietic stem and progenitor cell (HSPC) populations and technical limitations to isolate single clones from patients present major challenges to determine the impact of CALR mutant zygosity on clonal composition and diversity in MPN.

Human induced pluripotent stem (iPS) cells are primary cell lines with self-renewal capacity and have the potential to differentiate into any cell type of the three germ layers (Takahashi et al., 2007). Of note, iPS cells can be efficiently differentiated into HSCs allowing in vitro hematopoiesis modelling (Sugimura et al., 2017). Patient-derived iPS cells can be further genetically engineered with the CRISPR/Cas9 technology to repair or introduce diseaserelated mutations in the patient's genetic background. This approach provides a valuable tool to study genotype-phenotype correlations at the clonal level.

Thus, to overcome the aforementioned limitations, we generated MPN patient-specific iPS cell clones carrying homozygous or heterozygous $C A L R$ mutations or its isogenic unmutated counterpart. CALR mutations accelerated megakaryopoiesis independent of TPO, especially in homozygous mutant clones, and repair of the mutation using CRISPR/Cas9 reversed the effect. RNAseq of CALR-mutated vs. non-mutated MKs demonstrated increase in hypoxia signaling and leptin expression providing a better understanding of disease biology in ET and PMF and potential novel therapeutic targets.

\section{Results}

\section{Generation of MPN patient-derived iPS cell clones and repair of homozygous CALRins5 and del52 mutations}

iPS cells were established by reprogramming of peripheral blood mononuclear cells (PBMCs) from three MPN patients carrying CALRdel52, ins5, or del31 mutations (Table S1) and two healthy donors (HD). We obtained 9 homozygous and 55 heterozygous CALRdel52 iPS cell clones derived from a PMF patient, and 74 homozygous and 27 heterozygous CALRins5 clones from a post ET-MF patient (Table S1). Additionally, 64 heterozygous and 5 WT clones were generated from a PMF patient carrying a CALR del31 mutation (Table S1). Pluripotency 
of iPS cells was confirmed by positive staining for OCT3/4 and TRA-1-60 pluripotency markers (Fig. 1 A). Normal karyotype of MPN patient-specific iPS cell clones was shown by GTGbanding (Fig. S1 A). Endogenous expression of mutant CALR was confirmed by CALR allelespecific RT-qPCR and immunofluorescence (Fig. 1, B and C).

Reprogramming of CALRins5 and del52 PBMCs only resulted in homozygous and heterozygous clones but not CALR unmutated clones, and thus, CALRins5 and CALRdel52 mutations were repaired by CRISPR/Cas9 editing to obtain unmutated CALR iPS cells. Specific guide RNAs and CALR wildtype donor templates with homology arms were designed (Table S2 and S3). Successful correction of CALR mutation in homozygous clones was confirmed by PCR and subsequent Sanger sequencing (Fig. S1, B and C). For CALRdel52 mutation, three CRISPR clones out of 82 screened clones, and for CALRins5, two clones out of 88 showed a homozygous repair, amounting to a CRISPR efficiency of $3.37 \%$ and $2.27 \%$, respectively. No off-target effects introduced by CRISPR/Cas9 were found (Fig. S1, D and E). Loss of CALR mutant protein was verified by Western blot analysis in the CRISPR-engineered wild-type $\left(\mathrm{WT}_{\mathrm{cr}}\right)$ clones (Fig. $\left.1 \mathrm{D}\right)$. However, clones $01 \mathrm{WT}_{\mathrm{cr}}$ and $02 \mathrm{WT}_{\mathrm{cr}}$ of the CALRdel52 CRISPR approach showed unspecific bands when incubating with CALR mutated antibody.. To preclude that truncated versions of CALR protein resulted after the CRISPR repair, we performed standard amplification of the respective genomic region of the CALR gene, Sanger sequencing as well as next generation sequencing (NGS), and absence of mutated genomic $C A L R$ sequence was confirmed.

Furthermore, the presence of additional MPN-related mutations in all generated iPS cell clones was evaluated by NGS using a defined panel of MPN target genes (Kirschner et al., 2018). Besides the CALR mutations, no other clinically relevant MPN-related mutation was found in the generated patient-specific and HD control iPS cell clones (Table S4).

\section{MPO deficiency is detected uniquely in homozygous CALR-mutated iPS cell clones and is restored in repaired CALR clones}

Patient-derived iPS cells harboring the disease causing mutation(s) allow disease modelling in the culture dish. Homozygous CALRins5 mutations lead to myeloperoxidase (MPO) deficiency in patients (Theocharides et al., 2016), and we recapitulated this feature in vitro by differentiating our iPS cells towards hematopoietic cells in a modified embryoid body (EB)based protocol (Fig. 2 A) (Kovarova and Koller, 2012). We observed MPO deficiency in iPS cell-derived CD15+ cells harboring homozygous CALRins5 or CALRdel52 mutations. A significantly smaller CD15+cyMPO+ (cytoplasmic MPO) population was found in cells carrying homozygous CALRdel52 and CALRins5 mutations (Fig. 2, B and C). Meanwhile, relative MPO mRNA expression in homozygous CALR cells was upregulated (Fig. $2 \mathrm{D}$ ), confirming the posttranscriptional defect (Theocharides et al., 2016). Furthermore, a severe reduction of MPO 
activity in the homozygous CALR mutated cells was confirmed by cytochemical staining (Fig. $2 \mathrm{E})$. Most importantly, MPO activity was rescued by the correction of homozygous CALR mutations as shown for the $\mathrm{WT}_{\mathrm{cr}}$ clones, proving that homozygous mutant CALR is responsible for this defect.

\section{iPS cells harboring CALR mutations give rise to multiple myeloid cell lineages with stronger proliferative capacity}

Next, we aimed at getting further mechanistical insights into CALR mutations impacting hematopoietic cells. Therefore, iPS cells that carry the most common type 1 and type 2 CALR mutations, CALRdel52 and CALRins5, respectively, were differentiated towards the myeloid lineage with a modified differentiation protocol designated here as "spin-EB" differentiation (Fig. 3 A) (Liu et al., 2015). From day 8 onwards, hematopoietic cells were released from the EBs into suspension, with an increase of released cells until day 14, as shown in representative images of the differentiation. Homozygous CALRins5- and del52-mutant clones produced a significantly higher number of suspension cells compared to $\mathrm{WT}_{\mathrm{cr}}$ cells on day 14 (Fig. $3 \mathrm{~B}$ ). Additionally, cells bearing a heterozygous CALRdel52 mutation also showed an elevated number of suspension cells than $\mathrm{WT}_{\mathrm{cr}}$ cells. These data demonstrate that CALRins5 and CALRdel52 mutations enhance HSPC proliferation.

Flow cytometry analysis on day 10,12 and 14 of suspension cells confirmed generation of CD34+CD45+ HSPCs (Fig. 3 C; S2, A and B). Noteworthy, homozygous and heterozygous CALRdel52 mutated iPS cells, but not $\mathrm{WT}_{\mathrm{cr}}$ iPS cells, showed a stable level of HSPCs over the analyzed days. In $\mathrm{WT}_{\mathrm{cr}}$ cells, a significant reduction of HSPCs over the iPS cell differentiation time was observed accompanied by an overall higher number of HSCs generated on day 10 compared to hetero- and homozygous mutant cells.

To study the myeloid progenitor potential of iPS cell-derived HSCs, we performed a colonyforming-unit assay using CD34+ cells isolated on day 14 of differentiation, previously cultured in the presence of TPO. The heterozygous mutant CALRins5 CD34+ cells produced more colonies compared to $\mathrm{WT}_{\mathrm{cr}}$ cells (Fig. S2 C). The same trend was observed for the homozygous CALRins5- and CALRdel52 cells compared to control ( $p=0.0898$ and $p=0.0851$, respectively). To examine whether CALR mutation impacts on the expansion of CD34+ cells, expression of the proliferative marker Ki67 was evaluated in RT-qPCR (Figure S2D). We found that HSCs harboring a heterozygous CALRins5 mutation showed higher Ki67 expression (although not statistically significant, $p=0.1411$ ) compared to $\mathrm{WT}_{\mathrm{cr}} \mathrm{HSPCs}$. Together, those data confirmed a stronger amplification of cells in the CALR-mutated background, as described above. Identities of CFU-E (erythrocytic cells), CFU-M (macrophages), CFU-G (granulocytes) and CFU-GM (granulocytes/macrophages) were determined by morphological appearance and confirmed by QuikDiff staining (Fig. 3 D). HSPCs from all analyzed clones gave rise to 


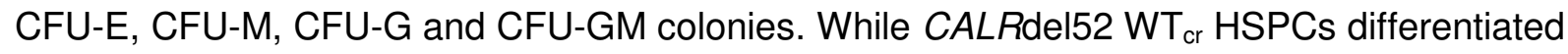
into the highest number of CFU-M, they showed less CFU-E. An increased number of erythrocytic colonies were found in CALRins5 heterozygous clones.

In summary, CALR mutants accelerate cell proliferation and CALR-mutated iPS cells show constant production of HSPCs.

\section{CALR-mutated iPS cells exhibit enhanced megakaryopoiesis and accelerated maturation in a TPO independent manner}

Aberrant megakaryopoiesis is the major hallmark of CALR-mutated MPN patients as well as in established mouse models. Therefore, we studied the impact of CALR-mutant zygosity on megakaryopoiesis in our patient-specific iPS cell model in our EB differentiation protocol (Fig. 3 A) (Liu et al., 2015). We found that iPS cells carrying homozygous or heterozygous CALRins5 and CALRdel52 mutation gave rise to significantly more $\mathrm{CD} 41+\mathrm{CD} 42 \mathrm{~b}+$ mature $\mathrm{MKs}$ compared to their $\mathrm{WT}_{\text {cr }}$ counterparts (Fig. $4 \mathrm{~A}$ ). Moreover, no difference in MK numbers obtained from HD or WT $_{\text {cr }}$ iPS cells was observed, demonstrating that the enhanced megakaryopoiesis is due to the CALR mutation. Mature megakaryocytes with typical multilobular nuclei and slight basophilic cytoplasm were detected in cytospins generated on day 14 of differentiation (Fig. 4 B). Of note, MKs carrying homozygous CALR mutation presented variable sizes, indicative of rapid maturation of aberrant MKs.

Mutant CALR binds to and activates the TPO receptor. To further study the impact of TPO on MK development in our iPS cell model, we differentiated the cells with and without supplementation of TPO (Fig. $4 \mathrm{C}$ ). In HD control cells as well as in $\mathrm{WT}_{\mathrm{cr}}$, we found a significantly lower number of MKs in the absence of TPO. In contrast, the number of iPS cellderived MKs harboring a CALR mutation was equal regardless of $C A L R$ mutation zygosity and the presence or absence of TPO. Thus, our CALR-mutated iPS cell-model recapitulates a key pathological feature of TPO-independent megakaryopoiesis.

By following CALR-mutated megakaryopoiesis in vitro, we observed a striking increase in immature CD41+CD61+ MKs in comparison to unmutated cells combining flow cytometry data from day 10, 12 and 14 (Fig. 4 D). We further analyzed the portion of mature MKs in the CD41+CD61+ cell population to analyze differences in the MK maturity level along the differentiation. A more in-depth analysis revealed less CD42b+CD41+CD61+ mature MKs from CALRdel52 $\mathrm{WT}_{\mathrm{cr}}$ iPS cells when compared to their homozygous counterparts on day 10 of differentiation (Fig. $4 \mathrm{E}$ ). The same was observed for the heterozygous CALRins 5 clone and with a tendency $(p=0.1794)$ also for homozygous $C A L R$ ins5-mutated MKs. However, on day 14 all genotypes showed the same MK maturity. Altogether, these data demonstrate an accelerated MK maturation process in CALR-mutated cells. 
MKs arise from progenitors shared with the erythrocytic lineage, the megakaryocyteerythrocytic progenitors (MEP). Therefore, we analyzed the proportion of erythrocytic cells (CD235a+CD45-) of the day 14 population (Fig. S2 E). HD control iPS cells and iPS cells with CALRdel52 background originated in an extremely low level of erythrocytic cells. However, CALRins5-mutated cells showed an increase in the erythrocytic population with a remarkable increase in the $\mathrm{WT}_{\mathrm{cr}}$ cells. These data demonstrate that the repair of the CALR mutation in the homozygous CALRins5-mutated cells led to enhanced erythropoiesis, which was not observed in HD control iPS cells or in the $\mathrm{WT}_{\mathrm{cr}}$ of $C A L R$ del52 cells. Based on this, we hypothesized that differentiation properties switch upon the repair of the CALR mutation from the megakaryocytic to the erythrocytic lineage, at least in the patient-specific background of our CALRins5-repaired clones.

\section{$C A L R$ mutation causes upregulation of MK-related genes in iPS cell-derived MKs}

MK development is subject to a highly coordinated process of megakaryocyte-specific gene expression and simultaneous prevention of erythrocytic development. To examine whether CALR-mutated and -unmutated MKs show differences at the transcriptional level, we analyzed gene expression of typical megakaryocytic genes and related transcription factors. Our clonal approach using iPS cell-derived CALR mutant MKs and their repaired counterparts are especially suitable for the analysis of solely mutated CALR-related changes.

To exclusively analyze the megakaryocytic subpopulation, CD61+ MKs were purified by magnetic activated cell sorting on day 14 of "spin-EB" differentiation. We compared the differential expression signatures of MKs within the group of CALRins5 MKs and CALRdel52 MKs in a heat map with unsupervised clustering (Fig. 5A, and B). We found that gene expression profiles of MKs with heterozygous or homozygous CALRins5 mutation built a welldefined cluster compared to unmutated MKs. Of note, heterozygous CALRins5-mutated MKs showed a stronger upregulation of MK-related genes compared to the homozygous CALRins 5 mutated MKs. Focusing on genes known to be involved in megakaryocytic development, we found that the expression of MPL was slightly enhanced in CALRins5 heterozygous clones and homozygous clones ( $p=0.0669$, Fig. $5 \mathrm{C}$ ) compared to $\mathrm{WT}_{\mathrm{cr}} \mathrm{MKs}$. Moreover, the early transcription factor FLI1 was highly upregulated in CALRins5 heterozygous and homozygous MKs compared to $\mathrm{WT}_{\mathrm{cr}} \mathrm{MKs}$ ( $p=0.0106$ and $p=0.051$, respectively). On the other hand, NFE2, an essential marker for terminal MK maturation involved in platelet release and expression of $V W F$, was strongly expressed in both homozygous and heterozygous CALRins5-mutated MKs (Tijssen and Ghevaert, 2013). Given that homozygous CALRins5-mutated MKs showed an upregulation of mature MK markers (NFE2 and VWF), this suggests enhanced megakaryopoiesis of homozygous mutant cells. In the iPS cell-derived HSPCs, we tested the 
expression of Ki67 in MKs, showing equal expression in both mutated CALRins5 and CALRdel52 and unmutated MKs (Fig. S2 F).

Unsupervised clustering of MKs with CALRdel52 mutation did not resultin clear clusters of each zygosity as given for CALRins5-mutated MKs (Fig. 4 B). Nonetheless, gene expression of mutated MKs, both heterozygous and homozygous, clustered closer together than $\mathrm{WT}_{\mathrm{cr}}$ MKs. In line with the expression data of CALRins5-mutated MKs, the strongest upregulation of MK related genes was found in heterozygous clones, suggesting an underlying mechanism including both mutated and WT protein, which enhances transcription of megakaryocytic genes, likely involving CALR chaperone function.

\section{CALR-mutated MKs exhibit higher granularity}

During megakaryopoiesis, MKs increase in size, produce alpha and dense granules, and develop a demarcation membrane system (DMS), the plasma membrane for future platelets (Ru et al., 2015). Terminally differentiated MKs form cytoplasmic protrusions, called proplatelets, which further mature into platelets with open canalicular systems (OCS) (Escolar and White, 1991).

To study in more detail whether structural features of MK maturation are present in our iPS cell-derived MKs, we captured ultrastructural transmission electron microscopy (TEM) images of CD61+ MKs on day14 of "spin-EB" differentiation for each CALRins5 genotype. TEM images of MKs with different genetic background revealed typical megakaryocytic structures including granules, DMS, pro-platelet protrusions and OCS (Fig. 6 A). Additionally, we determined the area of each MK from the TEM images of each CALR genotype (Fig. $6 \mathrm{~B}$ ). $\mathrm{WT}_{\text {cr }}$ MKs showed a slight increase $(p=0.1679)$ in size compared to homozygous mutant MKs. This supported our previous observation, that some small MKs were found in the population of homozygous mutant MKs in cytospin images (Fig. 4 B). It is reported that in vitro generated MKs undergo different stages of maturation, identifiable by the granularity of the cells defined by side scatter (SSC) in flow cytometry (Sim et al., 2017). Therefore, iPS-derived CD42b+CD41+CD61+ MKs were analyzed for their MFI of SSC. We found that the granularity of MKs is significantly enhanced in the homozygous CALRins5 MKs compared to the unmutated counterparts (Fig. $6 \mathrm{C})$, confirming that homozygous CALRins5-mutated MKs showed higher level of maturation compared to the unmutated MKs. No differences in the granularity of CALRdel52-mutated MKs was observed, again suggesting that type 1 and type 2 CALR mutations may not induce identical functional changes, as is also suggested by differences in their clinical profile.

In the following, we analyzed the distribution of CALR WT and mutant protein in our iPS cellderived MKs by immunofluorescence and confocal microscopy (Fig. 6 D; S3 A). We were able to identify CD42b positive MKs with pronounced poly-lobulated nuclei in mutated and 
unmutated MKs, highlighted in z-stacks of homozygous CALRins5 and WT MKs (Fig. S3 A). WT and mutated CALR protein were not differentially distributed in the MKs, and showed a more diffuse distribution in bigger MKs (yellow arrows). However, smaller MKs with less pronounced cytoplasm, showed a clustered localization of mutated CALR at the cell surface (green arrows). In some MKs, we were able to verify a co-localization of CALR protein and the endoplasmic reticulum (ER) (orange arrows).

\section{$C A L R$ mutation causes an upregulation of hypoxia related pathways in MKs}

Finally, to further unveil differences between RNA expression dependent on the zygosity of the CALR mutation, we performed RNA sequencing (RNAseq) analysis of day 14 CD61+ iPS cell-derived MKs. Three independent differentiation experiments were performed using either

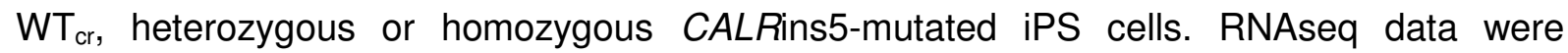
normalized using TMM normalization (Robinson and Oshlack, 2010) and subsequently voom transformed (Law et al., 2014).

Unsupervised clustering of differentially expressed genes (DEGs) was performed and depicted in a heat map (Fig. $7 \mathrm{~A}$ ). Samples efficiently clustered according to their CALR mutation zygosity, and higher similarity in the gene expression profile of mutated MKs was observed. Unsupervised sub-clustering of the heat map provided cluster 1 with mainly upregulated genes and cluster 2 with downregulated DEGs in homozygous mutant MKs. Gene ontology (GO) analysis of those clusters showed that MKs with homozygous CALR mutation upregulated interferon signaling and the response to hypoxia, while ECM organization was downregulated compared to $\mathrm{WT}_{\mathrm{cr}} \mathrm{MKs}$ as exemplarily shown for FN1 (Fig. 7 B). Of note, GO term including genes related to the ER were found to be affected in heterozygous and homozygous mutant MKs. The observed upregulation of hypoxia signaling was further validated by analysis of Pathway Responsive Genes (PROGENy) (Fig. 7 C) (Schubert et al., 2018). Moreover, the known hypoxia related gene NDRG1 was significantly upregulated in both heterozygous and homozygous CALRins5-mutated MKs compared to WT. (Fig. 7 D). Interestingly, PROGENy analysis showed that PI3K signaling was significantly downregulated in both heterozygous and homozygous mutant MKs compared to WT, similar to TGFb and EGFR pathways, which were downregulated in the homozygous mutant MKs compared to control MKs. In addition, a high number of DEGs were found by comparing different genotypes (Fig. S5). Genes found to be significantly upregulated in both heterozygous and homozygous mutant $\mathrm{MKs}_{\mathrm{s}}$ compared to $\mathrm{WT}_{\mathrm{cr}}$ MKs, included $\mathrm{H} 3 \mathrm{C} 3, \mathrm{CTSF}$, and leptin. Partial validation of depicted genes was performed by RT-qPCR (Fig. 7 D; Fig. S5 D).

\section{Discussion}


In the present study, we generated patient-specific iPS cell lines harboring CALRdel52, ins5 or del31 mutations, and the iPS cell-based model was applied to study the impact of CALR mutant zygosity on hematopoietic and more precisely on megakaryocytic differentiation at the clonal level. Patient-derived iPS cells carrying heterozygous CALR mutations have been recently described, but detailed comparisons of type 1 and type 2 CALR mutations with focus on their zygosity and detailed characterization of derived disease-driving MKs are missing (Gomez Limia et al., 2018, 2017; Secardin et al., 2021; Takei et al., 2018). To obtain isogenic CALR-repaired clones for CALRins5 and del52-mutated iPS cells, CRISPR/Cas9 technology was used to correct the mutations. Until now, the repair of $C A L R i n s 5$ mutations was reported at a conference abstract (Wang et al., 2018), and a CRISPR/Cas9 approach was used to model CALR mutations in murine cell lines (Abdelfattah and Mullally, 2018). To our knowledge, the repair of CALRdel52 mutations in MPN-specific iPS cells was not reported, yet. The absence of further MPN-related mutations of clinical significance was confirmed by NGS in our $C A L R$-mutated iPS cell clones. Hence, our findings are solely attributed to the CALR mutations.

MPO deficiency was demonstrated in homozygous CALRins5 iPS cell-derived CD15+ cells and, for the first time, also in homozygous CALRdel52 cells. Loss of MPO activity in MPN patients carrying homozygous CALR mutations was described by Theocharides and colleagues (Theocharides et al., 2016), and we were able to restore MPO activity by CRISPR/Cas9 gene repair, demonstrating the recovery of the chaperon function of CALR. The rescue of CALR WT characteristics was underlined by the fact that $W_{c r}$ and HD control iPS cells generated equal levels of MKs. Importantly, the iPS cell-derived MKs exhibited structural features of primary MKs such as formation of DMS, granules, multi-lobulated nuclei and proplatelets.

Megakaryocytic hyperplasia was reported for all types of MPNs and represents a diagnostic criterion of the World Health Organization (Barbui et al., 2018; Swerdlow et al., 2008; Zingariello et al., 2020). Our data demonstrate that both CALRins5- and del52-mutated iPS cells gave rise to more MKs than $\mathrm{WT}_{\mathrm{cr}}$ and HD control iPS cells. In particular, homozygous mutant clones produced higher numbers of MKs followed by heterozygous clones. Thus, the mutated CALR phenotype demonstrated in our study recapitulates the phenotype found in MPN patients and the data of Kollmann and colleagues demonstrating that the ectopic expression of CALRdel52 or CALRins5 mutants in human CD34+ HSPCs lead to enhanced megakaryopoiesis and platelet production (Kollmann et al., 2017). Furthermore, knock-in mice expressing homozygous CALRins 5 or del52 mutations exhibited stronger ET- and PMF-related phenotypes over heterozygous CALR-mutant mice, demonstrating that zygosity of the CALR mutation has an impact on disease development (Benlabiod et al., 2020). Araki and colleagues 
described mutant CALR protein homomultimers that are presumed to bind and activate the TPO receptor (Araki et al., 2018). This observation is confirmed by our data as a strong bias towards the MK lineage and enhanced MK maturation was observed for homozygous CALR-mutated iPS cells. Hence, we assume that mutant CALR homomultimers are formed more excessively in homozygous clones than in heterozygous clones.

Importantly, we demonstrated in our human iPS cell-based model that the CALR mutation led to the amplification of the MK lineage in an TPO-independent manner while WT and HD control cells showed pronounced MK population only in the presence of TPO. Of note, additional treatment of TPO did not further increase MK generation of CALR-mutated cells. This might be due to the reported oncogenic interaction of the TPO receptor and mutant CALR before reaching the cell surface (Masubuchi et al., 2020; Pecquet et al., 2019), already sufficiently occupying the TPO binding site. However, Secardin and colleagues observed an increase of CFU-MKs, generated from iPS-cell-derived CALR-mutated CD34+ CD43+ progenitors, in the presence of TPO (Secardin et al., 2021). These differences may be clone-dependent or exemplifies differences between liquid cultures and collagen/fibrin-based medium.

The observed amplification of the MK lineage in CALR-mutated cells raises the question whether it relies on enhanced proliferation or accelerated maturation of the HSPCs or MKs. Immunohistochemical staining of MKs showed a higher expression of proliferative Ki67 compared to controls (Malherbe et al., 2016). However, stronger Ki67 gene expression was not confirmed in our iPS cell-derived CALR-mutated MKs, but in the CALRins5 CD34+ population. Here, we observed a higher number of colonies in the CFU assays for CALRins5-mutated cells and higher cell numbers on day 14 of differentiation in the $C A L R$-mutated background compared to unmutated cells. Those data demonstrate that $C A L R$ mutations (especially CALRins5) impact on proliferation capacity of immature hematopoietic cells and MK differentiation.

While CALR mutations did not impact on MK proliferation, we showed that homozygous and heterozygous $C A L R$ mutations induced an accelerated maturation of MKs. Surface expression of CD42b, which is a marker for megakaryocytic maturation (Sim et al., 2017), was elevated on mutated $\mathrm{MKs}$ in comparison to $\mathrm{WT}_{\mathrm{cr}}$ and $\mathrm{HD}$ control MKs at an early time point of differentiation. Furthermore, higher expression of NFE2 in homozygous and heterozygous CALRins5 MKs compared to $\mathrm{WT}_{\mathrm{cr}} \mathrm{MKs}$ was observed. Moreover, we found that CALRins5 homozygous mutated MKs exhibited a higher level of granularity compared to cells harboring unmutated or heterozygous CALRins5. These findings are supported by Sim et al, that identified different maturation stages in MKs corresponding to their granularity (Sim et al., 2017). Taken together, our data demonstrate that CALR mutations cause an accelerated megakaryopoiesis in iPS cell-derived MKs rather than affecting megakaryocytic proliferation. 
In future work, our model could be used to study the relationship of accelerated and enhanced megakaryopoiesis and platelet production/release in CALR-mutated MKs.

We confirmed the upregulation of typical megakaryocytic genes such as NFE2, FLI1 and VWF in our iPS cell-derived MKs by RT-qPCR analysis and proceeded to an in-depth analysis of the global gene expression profile by RNAseq. Recent studies reported on MPN-related transcriptome analysis of in vitro differentiated MKs from primary CALR-mutated CD34+ (EIKhoury et al., 2020) or single-cell RNAseq of primary CD34+ cells from MF patients with either JAK2V617F or CALR mutation (Psaila et al., 2020). In contrast to the first mentioned study, we were able to relate differences of the megakaryocytic transcriptome to the underlying $C A L R$ genotype by analyzing a clonal cell population. Therefore, our model with defined mutational background more precisely elucidates mechanisms of aberrant megakaryopoiesis in MPN.

Our RNAseq data identified elevated interferon signaling and leptin gene expression in CALRmutated MKs. Leptin functions as a hormone and adipokine predominantly expressed by adipocytes (Cava and Matarese, 2004). However, a role of leptin in proinflammatory processes was reported, as it is able to activate human peripheral blood $B$ cells to induce the secretion of cytokines such as TNF- $\alpha$, IL-6 and IL-10 via JAK2/STAT3 and p38MAPK/ERK1/2 signaling pathways (Agrawal et al., 2011). In liver fibrosis, leptin promotes the proliferation and secretion of ECM molecules in myofibroblasts (activated hepatic stellate cells) via the leptin receptor (Vivoli et al., 2016). Also, in BM fibrosis, leptin receptor-positive mesenchymal stromal cells were identified to be responsible for collagen fiber generation and deposition (Decker et al., 2017). Hence, it is conceivable that megakaryocyte-produced leptin may be implicated in myofibroblast formation in MPN and contribute to the inflammatory environment in the BM niche.

Of note, the expression of ECM proteins was downregulated in CALR-mutated MKs, compared to control. The composition of ECM in the BM is essential for megakaryocytic differentiation and maturation (Noetzli et al., 2019). Synthesis of ECM components in MKs was described to be regulated via autocrine TGF- $\beta 1$ signaling (Abbonante et al., 2016). Our PROGENy analysis revealed that TGF- $\beta$ signaling was significantly downregulated in homozygous $C A L R$-mutant MKs, which may explain the downregulation of ECM genes in CALR-mutated MKs. However, in the above-mentioned study (Abbonante et al., 2016), in vitro experiments of CD34+-derived MKs from JAK2V617F-mutated PMF patients were performed, showing a stronger activation of TGF- $\beta$ signaling and, thus, increased expression of ECM components such as fibronectin and type IV collagen compared to control. However, the allele burden of the JAK2V617F mutation in the bulk of primary CD34+ cells was not specified. In contrast, ECM gene expression in our clonal iPS cell-derived MKs was solely correlated to the CALR mutation. 
In addition, the hypoxia signaling pathway was strongly upregulated in CALR-mutated vs unmutated MKs. In solid tumors, it is well established that hypoxia represents a key feature for tumor survival and development (Thomlinson and Gray, 1955), and the BM stem cell niche represents an environment of relatively low oxygen level (Irigoyen et al., 2017). The major regulator of cellular response to hypoxia is the hypoxia-inducible factor 1 (HIF-1a) (Semenza, 2009). Importantly, HIF-1a promotes survival of JAK2V617F-positive cells and was discovered to be a promising therapeutic target (Baumeister et al., 2020). Moreover, elevated protein levels of HIF-1a were recently described in blast phase MPN samples, suggesting a role of HIF-1a in MPN disease progression (Marinaccio et al., 2021). In addition, El-Khoury et al identified hypoxia as a deregulated pathway in primary CD34+ cells differentiated towards MKs from MPN-patients (El-Khoury et al., 2020). Hence, our data support the notion that hypoxiarelated factors may be promising targets in CALR-mutated MPNs.

Collectively, our study describes a comprehensive iPS cell system for evaluating mutant $C A L R$-induced phenotypes in MPN with a clonal genetic background, which is an valuable new tool for clinical drug screening and development of personalized therapies. We identified accelerated megakaryopoiesis and the upregulation of leptin expression as well as hypoxia and interferon signaling pathways as potential pathogenic mechanisms in CALR-mutated MKs.

\section{List of abbreviations}

CALR, calreticulin

CFU, colony forming unit

DEGs, differentially expressed genes

DMS, demarcation membrane system

EB, embryoid body

ET, essential thrombocythemia

FCS, forward scatter

iPS, induced pluripotent stem

MF, myelofibrosis

MKs, megakaryocytes

MPL, thrombopoietin receptor

MPN, myeloproliferative neoplasm

MPO, myeloperoxidase 
bioRxiv preprint doi: https://doi.org/10.1101/2021.07.05.451130; this version posted July 5, 2021. The copyright holder for this preprint (which was not certified by peer review) is the author/funder, who has granted bioRxiv a license to display the preprint in perpetuity. It is made available under aCC-BY-NC-ND 4.0 International license.

OCS, open canalicular system

PBMCs, peripheral blood mononuclear cells

PMF, primary myelofibrosis

$\mathrm{PV}$, polycythemia vera

SSC, side scatter

TEM, transmission electron microscopy

TPO, thrombopoietin

HSPCs, hematopoietic stem and progenitor cells 


\section{Declarations}

\section{Ethics approval and consent to participate}

Peripheral blood mononuclear cells (PBMCs) were obtained from MPN patients carrying CALRdel52 or CALRins5 mutation at the Department of Hematology at Zurich University, and from a CALR del31 mutated MPN patient at the centralized Biomaterial Bank in Hospital RWTH Aachen, University. Healthy donor PBMCs were provided from the Transfusion Medicine in the University Hospital RWTH Aachen. All PBMCs were donated after written informed consent, as approved by the ethics committees in Zurich and the Medical Faculty of RWTH Aachen (EK127/12, EK 206/09, and EK099/14).

\section{Consent for publication}

All authors have signed the form of consent to publication.

\section{Competing interests}

SK reports funding from Novartis, Bristol-Myers Squibb, Janssen/Geron; advisory board honoraria from Pfizer, Incyte, Ariad, Novartis, AOP Pharma, BMS, Celgene, Geron, Janssen, CTI, Roche, Baxalta, Sanofi; patent for BET inhibitor at RWTH Aachen University; honoraria from Novartis, BMS, Celgene, Geron, Janssen, Pfizer, Incyte, Ariad, Shire, Roche, AOP Pharma; and other financial support (e.g. travel support) from Alexion, Novartis, BMS, Incyte, Ariad, AOP Pharma, Baxalta, CTI, Pfizer, Sanofi, Celgene, Shire, Janssen, Geron, Abbvie, Karthos. 


\section{Material and methods}

\section{Generation of MPN patient-specific and healthy donor iPS cells}

Peripheral blood mononuclear cells (PBMCs) were obtained from MPN patients carrying a CALRdel52 or CALRins5 mutation at the Department of Hematology at Zurich University, and from a CALRdel31 mutated MPN patient at the centralized Biomaterial Bank in Hospital RWTH Aachen, University. HD PBMCs were provided from the Transfusion Medicine in the University Hospital RWTH Aachen. All PBMCs were donated after written informed consent, as approved by the ethics committees in Zurich and the Medical Faculty of RWTH Aachen. PBMCs were reprogrammed using a CytoTune iPS 2.0 Sendai Reprogramming Kit (Thermo Fisher Scientific, Waltham, MA; USA) according to the manufacturer's instructions. After reprogramming, individual iPS cell colonies were picked and screened for CALR genotypes by PCR or allele-specific PCR.

\section{Next generation sequencing}

For the detection of mutations from gDNA by next generation sequencing (NGS), two panels individually designed and validated for routine hematology diagnostic were used. Either $250 \mathrm{ng}$ or 75ng of gDNA were used for library preparation with either a Truseq Custom Amplicon Kit (Illumina, San Diego, USA) or an Ampliseq for Illumina Custom Panel (Illumina) covering the relevant regions of either 31 (ABL1, ASXL1, BARD1, CALR, CBL, CHEK2, CSF3R, DNMT3A, ETNK1, ETV6, EZH2, IDH1, IDH2, JAK2, KIT, KRAS, MPL, NFE2, NRAS, PDGFRA, PTPN11, RUNX1, SETBP1, SF3A1, SF3B1, SH2B3 (LNK), SRSF2, TCF12, TET2, TP53, U2AF1)(Kirschner et al., 2018) or 32 genes (ABL1, ASXL1, BRAF, BTK, CALR, CBL, CSF3R, CXCR4, DNMT3A, ETNK1, EZH2, FLT3, IDH1, IDH2, JAK2, KIT, KRAS, MPL, MYD88, NFE2, NPM1, NRAS, PTPN11, RUNX1, SETBP1, SF3B1, SH2B3, STAT5B, TET2, TP53, U2AF1, WT1) associated with hematologic malignancies. The final libraries were sequenced with 2x250bp on a MiSeq (Illumina). The MiSeq onboard software was used (Real time analysis software v1.18.54, Illumina) for demultiplexing and FastQ file generation. Alignment and variant calling were performed with the SeqNext-Module of the SeqPilot-Software (JSI medical systems, Version 4.4.0 Build 509). Variants were called with a bidirectional frequency of $>5 \%$ (JAK2 V617F and KIT D816V >1\%) and reviewed manually.

\section{Cytogenetic analysis}

Patient-derived iPS cell clones were seeded on matrigel coated $25 \mathrm{~cm}^{2}$ cell culture flasks. Normal chromosomal constitution was verified by conventional karyotyping of iPS cell clones by means of GTG banding at 400 to 550 band level. Metaphase spreads were prepared using standard procedures of blocking cell division at metaphase, hypotonic treatment, and methanol/acetic acid fixation (3:1). The banding techniques included the use of a trypsin 
pretreatment (GTG-banding) carried out according to standard protocols. Microscopy was performed with Axioplan fluorescence microscope (Carl Zeiss) and IKARUSTM digital imaging systems (MetaSystems, Altlussheim, Germany). An average of 20 mitoses were analyzed for each clone.

\section{Immunofluorescence staining}

iPS cell colonies were cultured on Matrigel coated coverslips in 4-well-plates. Cells were fixed with $4 \%$ paraformaldehyde (Sigma Aldrich) and blocked with goat serum (Merck Millipore, Darmstadt, Germany). In order to detect the expression of pluripotent markers and mutant CALR, cells were incubated with following primary antibodies at $4{ }^{\circ} \mathrm{C}$ overnight: TRA-1-60 (Merck Millipore), OCT3/4 (H-134, Santa Cruz, CA, USA) or mouse monoclonal antibody CAL2 (Dianova, Hamburg, Germany). Cells were washed three times with PBS on the next day and incubated with corresponding secondary antibodies goat anti-rabbit $\lg G(H+L)$ FITC (Thermo Fisher Scientific), goat anti-mouse IgM (H) Alexa Fluor 594 (Thermo Fisher Scientific) or goat anti-mouse IgG Alexa Fluor 647 (for CALR mutant, clone poly4053, BioLegend, San Diego, $\mathrm{CA}, \mathrm{USA}$ ) for $1 \mathrm{~h}$ in the dark. Nuclei were stained with DAPI (Vector Laboratories, Burlingame, CA, USA) and coverslips were mounted with Dako Fluorescence Mounting Medium (Dako, Jena, Germany). Fluorescent image analysis was performed using an Axiovert 200 microscope (Carl Zeiss, Jena, Germany) and IPLab Spectrum software (BD, Franklin Lakes, USA).

In order to detect localization of WT and mutand CALR in MKs, cells were fixed with 3,7\% PFA, washed with PBS and permeabilized with 0,1\% Trition-X and 2,5\% BSA. Afterwards cells were incubated with detection reagent for ER-staining and Hoechst (Enzo Life Sciences, Farmingdale,USA ) for $20 \mathrm{~min}$ at $37^{\circ} \mathrm{C}$. Cells were washed with PBS and blocked wit $5 \%$ BSA in PBS. Cells were incubated with the following primary antibodies at $4{ }^{\circ} \mathrm{C}$ overnight: polyclonal antibody CD42b (Thermo Fisher Scientific) and mutant CALR antibody (Dianova) or WT CALR monoclonal antibody (Novus, Littleton, USA). On the next day, cells were washed with PBS and incubated with corresponding secondary antibodies goat anti-rabbit IgG Alexa Fluor 555 $(\mathrm{H}+\mathrm{L})$ and goat anti-mouse $\lg \mathrm{G}(\mathrm{H}+\mathrm{L})$ Alexa Fluor 647(all Thermo Fisher Scientific) for $1 \mathrm{~h}$ at RT. Coverslips were mounted with Fluorescence Mounting Medium (Dako, Carpinteria, USA). Fluorescent image analysis was performed using a confocal Scanning Microscope (LSM 710) microscope and Zeiss 2012 software (both Carl Zeiss, Jena, Germany).

\section{RNA isolation and qPCR}

RNA of iPS cells and iPS-cell derived CD34+ HSCS and CD61+ MKs was isolated using the RNeasy Mini Kit (QIGAEN GmbH, Hilden, Germany) according to manufacturer's protocol. RNA (500 ng) was used for cDNA synthesis. Quantitative RT-PCR was performed using the 7500 Fast Real-time PCR System (Applied Biosystems by Life technologies, Paisley, UK) with 
the SYBR Select Master Mix for CFX (Applied Biosystems). The sequences of primers used for-qPCR are listed in Table S7. All primers were purchased from Eurofins-MWG biotech (Ebersberg, Germany). The mRNA expression level of the target gene is determined in \% of GAPDH or MT-ATP6.

\section{SDS-Page and Western blot}

SDS-Page and Western blot analysis were conducted as previously described (Han et al., 2016). Primary and secondary antibodies are listed in Table S6.

\section{CRISPR/Cas9n-mediated repair of homozygous CALRins5 and CALRdel52 mutation in iPS cells}

The Alt-R ${ }^{\mathrm{TM}}$ CRISPR-Cas system (Integrated DNA Technologies IDT, Coralville, USA) was used to efficiently correct homozygous CALRins5 or CALRdel52 mutations in MPN patient-specific iPS cells. For precise editing the Alt-R® S.p HiFi Cas9 Nuclease V3 was used together with a single-stranded donor template to repair the double strand break by the cellular repair mechanisms of homology directed repair (HDR). In brief, guide RNA composing of crRNA and tracrRNA was combined with HiFi. Cas9 Nuclease to assembly the CRISPR-Cas9 ribonucleoprotein complex. Singularized iPS cells were gently mixed with the ribonucleoprotein complex, single-stranded donor template, and electroporation enhancer. Nucleofection was performed using the 4D NucleofectorTM X-Unit and the P3 Primary Cell 4D-NucleofectorTM $X$, Kit S (both from Lonza, Basel, Switzerland). HDR was stimulated by HDR enhancer (IDT, Coralville, USA). After nucleofection, iPS cells were seeded on previously coated Laminin 521 (Biolamina, Sundbyberg, Sweden) plates to support cell viability in StemMACS ${ }^{\mathrm{TM}}$ iPS-Brew XF (Miltenyi Biotec, Bergisch Gladbach, Germany) supplemented with $10 \mu \mathrm{M}$ of Rock inhibitor Y-27632 and 1x CloneR (Stemcell Technologies). Repair of CALRins5 and CALRdel52 mutation was verified by allele-specific or flanking PCR, respectively, and proofed by Sanger sequencing and Western blot. Possible off-target effects were excluded by PCR reaction of off-target sites and subsequent Sanger sequencing. Sequences of crRNA, donor template and primers are provided in Table $\mathrm{S} 2$ and 3.

\section{Differentiation of iPS cells into hematopoietic progenitors and myeloid subsets with the} "EB-based" protocol

iPS cells were cultured on irradiated mouse embryonic fibroblast feeder layer in KnockOutDMEM based iPS cell medium. More detailed information regarding cell culture conditions can be found in Table S8.

For the generation of iPS cell-derived CD34+ HSCs, CALR iPS cells were subjected to mesoderm commitment and hematopoietic differentiation with an embryonic body (EB)-based 
protocol modified from the differentiation method described by Kovarova and colleagues (Kovarova and Koller, 2012). CD34+ HSCs were cultured and differentiated into myeloid subsets in the StemPro 34 SFM medium (Thermo Fisher Scientific) based progenitor medium at the maximum density of $1 \times 10^{6}$ cells $/ \mathrm{ml}$. Details of EB and HSCs differentiation media are provided in Table S8.

\section{Cell morphology analysis}

Phase contrast images of iPS cell generation and differentiation were obtained with EVOS FL microscope (Thermo Fisher Scientific). To characterize the morphologies of iPS cell-derived precursors and myeloid subsets, cells were spun onto slides with Shandon Cytospin 4 cytocentrifuge (Thermo Fisher Scientific) and stained with Diff Quik solution 1 and 2 (Medion Diagnostics, Düdingen, Switzerland) after methanol fixation. Images were acquired using a Leica DMRX microscope and Leica Application Suite software (Leica Microsystems).

\section{MPO cytochemical staining}

In order to evaluate MPO functional activity, iPS cell-derived hematopoietic cells at differentiation day 15 were centrifuged onto slides and fixed with LEUCOGNOST® Fixing Mixture. The fixed cells were stained with LEUCOGNOST® POX Kit according to the manufacturer's instructions. Counterstaining of nucleus was performed using Mayer's hemalum solution and slides were mounted with Kaiser's glycerol gelatin (the Kit and all reagents were obtained from Merck Millipore). Images were acquired as described above.

\section{Differentiation of iPS cells into hematopoietic stem cells and myeloid subsets with the “spin-EB" protocol}

Feeder-free iPS cell culture was maintained on matrigel coated 6-well plates and routinely passaged with $1 \mathrm{ml}$ accutase or $0.5 \mathrm{mM}$ EDTA (Thermo Fisher Scientific). To enhance single cell survival, $10 \mu \mathrm{M}$ ROCK inhibitor Y-27632 was added to the maintenance culture medium StemMACs iPS Brew XF for $24 \mathrm{~h}$ after seeding. iPS cell medium was changed daily.

Human iPS cells were differentiated into HSPCs, MKs and erythrocytic cells adapted from the differentiation protocol by Liu et. al (Liu et al., 2015). Briefly, iPS cells were seeded into Ubottom-shaped 96 -well plates with a density of 3,000 cells/well in cytokine supplemented serum-free medium (SFM). To allow spheroid formation, plates were spun at $380 \mathrm{~g}$ for $5 \mathrm{~min}$. From day 2 to day 8, cells were cultured in SFM with $10 \mathrm{ng} / \mathrm{ml}$ VEGF (Miltenyi Biotec), $10 \mathrm{ng} / \mathrm{ml}$ BMP4 (Miltenyi Biotec), $10 \mathrm{ng} / \mathrm{ml}$ bFGF (Peprotech, Hamburg, Germany) and SCF $(0.5 \%$ supernatant of SCF secreting CHO KLS cells). From day 8 onwards, BMP4 and VEGF were removed from the medium. On day $11,20 \mathrm{ng} / \mathrm{ml}$ TPO (Miltenyi Biotec) was added to the medium. On day 14 , cells were harvested and filtered through a $100 \mu \mathrm{m}$ filter to separate the 
bioRxiv preprint doi: https://doi.org/10.1101/2021.07.05.451130; this version posted July 5, 2021. The copyright holder for this preprint

EBs from the suspension cells. Single cells were analyzed by flow cytometry and purified for CD61+ and CD34+ cell fractions. More detailed information on SFM Medium is given in Table S8.

\section{Flow cytometry analysis}

To evaluate cell surface progenitor and lineage specific markers, single cells were analyzed on day 10, day 12 and day 14 of "spin-EB" differentiation and on day 10 and day 15 of "EB-based" differentiation. Cells were harvested and passed through a $100 \mu \mathrm{m}$ cell strainer, centrifuged at $350 \mathrm{~g}$ for $5 \mathrm{~min}$. After washing with ice cold FACS buffer, single cells were incubated with lineage-specific antibodies at $4^{\circ} \mathrm{C}$ for $30 \mathrm{~min}$. Stained cells were resuspended in $300 \mu \mathrm{l}$ FACS buffer and analyzed on a FACS Canto II (BD). All staining and washing steps were performed in FACS buffer consistent of PBS supplemented with 2 mM EDTA and $2 \%$ BSA (PAN Biotech, Aidenbach, Germany).

To identify the intracellular MPO expression, differentiated hematopoietic cells at day 15 of "EB-based" differentiation were fixed and permeabilized using Fix \& Perm Cell Permeabilization Kit (Thermo Fisher Scientific). Cells were firstly stained for cell surface markers CD15 and CD45 for 20 min at room temperature (RT), followed by intracellular MPO staining for $20 \mathrm{~min}$ at RT after fixation and permeabilization. Stained cells were evaluated by Gallios flow cytometer (Beckman Coulter, Krefeld, Germany). All data were analyzed by FlowJo $^{\mathrm{TM}}$ (version 10, Oregon, USA).

Full list of antibodies is summarized in Table S5. In each experiment matched Ig isotype controls were used to set background fluorescence.

\section{Purification of CD34+ HSPCs and CD61+ megakaryocytes by magnetic activated cell sorting}

On day 25-35 of "EB-based" differentiation and on day 14 of "spin-EB" differentiation anti-CD34 and anti-CD61 MicroBeads (Miltenyi Biotec, Bergisch Gladbach, Germany) were used to separate HSPCs and MKS, respectively. Purification of cells was performed according to the protocol provided by the manufacturer. In brief, single cell suspension was incubated with CD61 or CD34 MicroBeads for 15 or $30 \mathrm{~min}$, respectively, to label the cells magnetically. Cell suspension was loaded onto a LS column installed in a magnetic field. After washing three times with MACS buffer consisting of PBS supplemented with $5 \%$ FCS (Thermo Fisher Scientic) and 2 mM EDTA to remove unbounded cells, the column was removed from the magnetic field and positively selected cell fraction was eluted with $5 \mathrm{ml}$ of MACS buffer from the column. After centrifugation at $300 \mathrm{~g}$ for $10 \mathrm{~min}$, purified cells were counted and further processed. CD61+ MKs and CD34+ HSPCs were either snap frozen for RT-qPCR or used for transmission electron microscopcy or seeded in Colony-Forming Unit Assay, respectively. 


\section{Colony-forming unit assay of CD34+ HSPCs}

Purified CD34+ cells were seeded in MethoCult ${ }^{\mathrm{TM}}$ (Stemcell Technologies) supplemented with $20 \mathrm{ml} \mathrm{IMDM}$ (Thermo Fisher Scientific), $50 \mathrm{ng} / \mathrm{ml}$ hSCF, $10 \mathrm{ng} / \mathrm{ml}$ hlL-3. $10 \mathrm{ng} / \mathrm{ml}$ hGMSCF and $14 \mathrm{ng} / \mathrm{ml}$ hEPO (all ImmunoTools Friesoythe; Germany) in a cell density of 5,000 cells/ml. After 10-12 days of culture at $37^{\circ} \mathrm{C}$, colonies were identified and counted based on morphology using light microscopy (Motic, Barcelona, Spain).

\section{Transmission electron microscopy}

iPS cell-derives CD61+ megakaryocytes at day 14 of "spin-EB" differentiation were prepared as described previously. Purified MKs were washed with PBS and fixed with $3 \%$ glutaraldehyde for at least $2 \mathrm{~h}$ at RT, followed by embedding in $5 \%$ low melting agarose (Merck). Gelatinated blocks were washed in $0.1 \mathrm{M}$ Soerensen's phosphate buffer (Merck) and post-fixed in $17 \%$ sucrose buffer (Merck) containing $1 \%$ OsO4 (Roth, Karlsruhe, Germany). Subsequently, specimens were dehydrated performing an ascending ethanol series repeating the last step for three times $(30,50,70,90$, and $100 \%$ ethanol; 10 min each step). Dehydrated samples were consecutively incubated in propylene oxide (Serva, Heidelberg Germany) for $30 \mathrm{~min}$, in a mixture of EPON resin (Serva) and propylene oxide (1:1) for $1 \mathrm{~h}$, and in pure EPON for $1 \mathrm{~h}$. EPON polymerization was conducted at $90^{\circ} \mathrm{C}$ for $2 \mathrm{~h}$. Ultrathin sections of $70-100 \mathrm{~nm}$ were cut with a Reichert Ultracut S ultramicrotome (Leica, Wetzlar, Germany) equipped with a diamond knife (Leica) and picked up on copper-rhodium grids (Plano, Wetzlar, Germany). Contrast was enhanced by staining with $0.5 \%$ uranyl acetate and $1 \%$ lead citrate (both EMS, Munich, Germany). Samples were viewed at an acceleration voltage of $60 \mathrm{kV}$ using a Zeiss Leo 906 (Carl Zeiss, Jena, Germany) transmission electron microscope. Image processing and analysis was performed using ImageJ (Schneider et al., 2012).

\section{Preparation of samples for RNA Sequencing}

RNA of purified CD61+ MKs was isolated as described in Supplementary Information. Quality was verified by TapeStation 4200 (Agilent, Santa Clara, USA) to determine RNA integrity number (RIN). RNA concentration was measured with Fluorometer Quantus ${ }^{\mathrm{TM}}$ (Promega, Fitchburg, USA). According to manufacturer's protocol, ribosomal RNA was depleted using

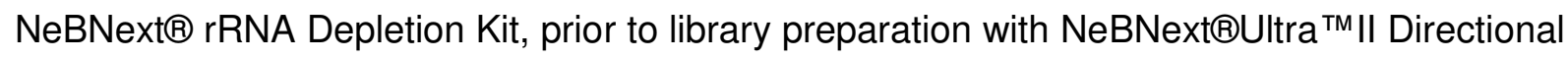
RNA Library Prep Kit for Illumina (both New England BioLabs, Ipswich, USA) using an RNA input of $100 \mathrm{ng}$ per sample (New England BioLabs, Ipswich, USA). Subsequently, samples were sequenced in paired end reads ( $2 \times 76$ bp, dual indexed) on two NextSeq High Output Kits 
v2.5 (150 cycles) on a NextSeq 500 instrument (both Illumina, San Diego, USA) to have sufficient reads for data analysis.

\section{Statistical analysis}

Graphical display and statistical analysis were performed with Prism 7 (GraphPad, San Diego, California, USA). Unless otherwise stated, all experiments were performed in triplicates. For each $C A L R$ genotype, two different clones were used, except for heterozygous CALRdel52 clone, where only one clone was generated after reprogramming. Comparisons among two groups were performed by using unpaired Student $t$ test. For multiple group comparisons, ANOVA with Bonferroni posttest was performed. $p$ values $<0.05$ were considered statistically significant $\left({ }^{*} p<0.05,{ }^{* *} p<0.01,{ }^{* * *} p<0.001\right)$.

\section{Online supplemental material}

Fig S1 A shows karyotype analysis of patient-derived iPS cell cones. Fig S1, B and C display the alignment of iPS cell clones after CRISPR/Cas9 repair of CALR-mutated maternal clone with CALR WT sequence. Fig. S1, $D$ and $E$ show sequencing analysis of potential CRISPR/Cas9 off-target products. Fig. S2, A and B show gating strategy of flow cytometry analysis exemplarily. Fig. S2 C shows number of colonies counted in a CFU assay, related to Fig. 3 D. Fig. S2 D shows Ki67 expression of CD34+ HSPCs. Fig. S2 E shows the quantification of erythrocytic cells analyzed by flow cytometry. Fig. S2 F demonstrates Ki67 expression of CD61+ MKs. Fig. S3, A and B show immunofluorescence analysis of mutant and WT CALR in iPS-cell derived MKs. Fig. S4 shows gene ontology (GO) analysis of clusters identified in a heat map of DEGs shown in Fig. 7 A. Fig. S5, A, B and C show volcano plots of DEGs compared among CALR genotypes. Fig. S7 D shows validation of genes identified in Fig. 7 D by RT-qPCR.

Table S1 summarizes details of reprogrammed patients' samples and generated iPS cell clones. Table S2 lists guide RNA used for CRISPR/Cas9 mediated CALR repair. Table S3 shows donor templates used for homology directed repair. Table S4 displays MPN-related mutations and polymorphisms of CALR iPS cell clones. Table S5 lists antibodies used for flow cytometry. Table S6 lists primary and secondary antibodies used for Western blot and Immunofluorescence staining. Table S7 summarizes Primers used for genotyping and RTqPCR. Table S8 summarizes media compositions.

\section{Acknowledgements}

We thank Reinhild Herwartz, Melanie Baumann and Lucia Vankann for technical assistance of MPO cytochemical staining and flow cytometry analysis, Ulla Gollan for cytogenetic analysis. We thank Sabrina Ernst, Carmen Schalla, and Prof. Dr. rer. nat. Gerhard Müller-Newen for technical support on immunofluorescence staining experiments. Biomaterial 
samples were provided by the RWTH centralized Biomaterial Bank Aachen (RWTH cBMB, Aachen, Germany) in accordance with the regulations of the biomaterial bank and the approval of the ethics committee of the medical faculty, RWTH Aachen. This work was in part supported by the Flow Cytometry Facility, a core facility of the Interdisciplinary Center for Clinical Research (IZKF) Aachen within the Faculty of Medicine at RWTH Aachen University. This work was supported by the Confocal Microscopy Facility, a Core Facility of the Interdisciplinary Center for Clinical Research (IZKF) Aachen within the Faculty of Medicine at RWTH Aachen University. The study was supported by the Genomics Facility, supported by a grant from the Interdisciplinary Center for Clinical Research within the Faculty of Medicine at RWTH Aachen University.

\section{Funding}

This work was supported in part by funds from the German Research Foundation (Deutsche Forschungsgemeinschaft DFG CH1509/1-1, KO2155/7-1, ZE432/10-1 and GE 2811/4-1) as part of the clinical Research Unit CRU344, a research grant from the Deutsche José Carreras Leukämie-Stiftung (DJCLS 16R/2017) to SK, and a research grant from the German Research Foundation (DFG KO2155/6-1) to SK. LH was supported by the National Natural Science Foundation of China (No. 82000133). MAST was financed by CAPES-Alexander von Humboldt postdoctoral fellowship (99999.001703/2014-05). AT was supported by the Prof. Dr. Max Cloëtta foundation. Parts of this work were generated within the $\mathrm{PhD}$ thesis project of $\mathrm{KO}$ and $\mathrm{LH}$.

\section{Author contributions:}

Conceptualization: K. Olschok, L. Han, M.A .S. Toledo, N. Chatain, and S. Koschmieder;

Data curation: M. Graßhoff, I.G. Costa, A. Maurer, and J. Baumeister;

Formal analysis: K. Olschok, L. Han, M. Graßhoff, I.G. Costa, A. Maurer, and J. Baumeister;

Funding acquisition: N. Chatain, and S. Koschmieder;

Investigation: K. Olschok, L. Han, M. A .S. Toledo, J. Böhnke, A. Maurer, H. M. Schüler, E. M. Buhl, K. Pannen, S. Gupta, and P. Boor;

Methodology: K. Olschok, L. Han, M. A. S. Toledo, and J. Böhnke

Project administration: K. Olschok, L. Han, M.A .S. Toledo, N. Chatain, and S. Koschmieder;

Resources: A. Theocharides, M. Kalmer, D. Gezer, and M. Zenke;

Software: M. Graßhoff, and I. G. Costa; 
Supervision: M .A .S. Toledo, N. Chatain, and S. Koschmieder;

Validation: K. Olschok, and L. Han;

Visualization: K. Olschok, L. Han, M. A. S. Toledo, and M. Graßhoff

Writing - original draft: K. Olschok, and L. Han;

Writing - review \& editing: K. Olschok, L. Han, M. A .S. Toledo, J. Böhnke, M. Graßhoff, I.G.

Costa, A. Theocharides, A. Maurer, H. M. Schüler, E. M. Buhl, K. Pannen, J. Baumeister, M.

Kalmer, S. Gupta, P. Boor, D. Gezer, T. H. Brümmendorf, N. Chatain, and S. Koschmieder;

All authors approved the final version of the manuscript.

Kathrin Olschok and Lijuan Han have contributed equally to this work.

Nicolas Chatain and Steffen Koschmieder share last authorship.

\section{Corresponding authors}

Correspondence to Nicolas Chatain and Steffen Koschmieder. 


\section{References}

Abbonante, V., Di Buduo, C.A., Gruppi, C., Malara, A., Gianelli, U., Celesti, G., Anselmo, A., Laghi, L., Vercellino, M., Visai, L., lurlo, A., Moratti, R., Barosi, G., Rosti, V., Balduini, A., 2016. Thrombopoietin/TGF- $\beta 1$ Loop Regulates Megakaryocyte Extracellular Matrix Component Synthesis. Stem Cells 34, 1123-1133. https://doi.org/10.1002/stem.2285

Abdelfattah, N.S., Mullally, A., 2018. Using CRISPR/cas9 gene editing to investigate the oncogenic activity of mutant calreticulin in cytokine dependent hematopoietic cells. J. Vis. Exp. 2018, 1-9. https://doi.org/10.3791/56726

Agrawal, S., Gollapudi, S., Su, H., Gupta, S., 2011. Leptin Activates Human B Cells to Secrete TNF- $\alpha$, IL-6, and IL-10 via JAK2/STAT3 and p38MAPK/ERK1/2 Signaling Pathway. J. Clin. Immunol. 31, 472-478. https://doi.org/10.1007/s10875-010-9507-1

Araki, M., Yang, Y., Imai, M., Mizukami, Y., Kihara, Y., Sunami, Y., Masubuchi, N., Edahiro, Y., Hironaka, Y., Osaga, S., Ohsaka, A., Komatsu, N., 2018. Homomultimerization of mutant calreticulin is a prerequisite for MPL binding and activation. Leukemia. https://doi.org/10.1038/s41375-018-0181-2

Araki, M., Yang, Y., Masubuchi, N., Hironaka, Y., Takei, H., Morishita, S., Mizukami, Y., Kan, S., Shirane, S., Edahiro, Y., Sunami, Y., Ohsaka, A., Komatsu, N., 2016. Activation of the thrombopoietin receptor by mutant calreticulin in CALR-mutant myeloproliferative neoplasms. Blood 127, 1307-16. https://doi.org/10.1182/blood-2015-09-671172

Barbui, T., Thiele, J., Gisslinger, H., Kvasnicka, H.M., Vannucchi, A.M., Guglielmelli, P., Orazi, A., Tefferi, A., 2018. The 2016 WHO classification and diagnostic criteria for myeloproliferative neoplasms: document summary and in-depth discussion. Blood Cancer J. 8, 15. https://doi.org/10.1038/s41408-018-0054-y

Baumeister, J., Chatain, N., Hubrich, A., Maié, T., Costa, I.G., Denecke, B., Han, L., Küstermann, C., Sontag, S., Seré, K., Strathmann, K., Zenke, M., Schuppert, A., Brümmendorf, T.H., Kranc, K.R., Koschmieder, S., Gezer, D., 2020. Hypoxia-inducible factor 1 (HIF-1) is a new therapeutic target in JAK2V617F-positive myeloproliferative neoplasms. Leukemia 34, 1062-1074. https://doi.org/10.1038/s41375-019-0629-z

Benlabiod, C., Cacemiro, M. da C., Nédélec, A., Edmond, V., Muller, D., Rameau, P., Touchard, L., Gonin, P., Constantinescu, S.N., Raslova, H., Villeval, J.-L., Vainchenker, W., Plo, I., Marty, C., 2020. Calreticulin del52 and ins5 knock-in mice recapitulate different myeloproliferative phenotypes observed in patients with MPN. Nat. Commun. 11, 4886. https://doi.org/10.1038/s41467-020-18691-3 
Campo, E., Swerdlow, S.H., Harris, N.L., Pileri, S., Stein, H., Jaffe, E.S., 2011. The 2008 WHO classification of lymphoid neoplasms and beyond: evolving concepts and practical applications. Blood 117, 5019-32. https://doi.org/10.1182/blood-2011-01-293050

Cava, A. La, Matarese, G., 2004. The weight of leptin in immunity. Nat. Rev. Immunol. 4, 371-379. https://doi.org/10.1038/nri1350

Chachoua, I., Pecquet, C., El-Khoury, M., Nivarthi, H., Albu, R.-I., Marty, C., Gryshkova, V., Defour, J.-P., Vertenoeil, G., Ngo, A., Koay, A., Raslova, H., Courtoy, P.J., Choong, M.L., Plo, I., Vainchenker, W., Kralovics, R., Constantinescu, S.N., 2016.

Thrombopoietin receptor activation by myeloproliferative neoplasm associated calreticulin mutants. Blood 127, 1325-35. https://doi.org/10.1182/blood-2015-11-681932

Decker, M., Martinez-Morentin, L., Wang, G., Lee, Y., Liu, Q., Leslie, J., Ding, L., 2017. Leptin-receptor-expressing bone marrow stromal cells are myofibroblasts in primary myelofibrosis. Nat. Cell Biol. 19, 677-688. https://doi.org/10.1038/ncb3530

El-Khoury, M., Cabagnols, X., Mosca, M., Vertenoeil, G., Marzac, C., Favale, F., Bluteau, O., Lorre, F., Tisserand, A., Rabadan Moraes, G., Ugo, V., Ianotto, J.C., Rey, J., Solary, E., Roy, L., Rameau, P., Debili, N., Pasquier, F., Casadevall, N., Marty, C., Constantinescu, S.N., Raslova, H., Vainchenker, W., Plo, I., 2020. Different impact of calreticulin mutations on human hematopoiesis in myeloproliferative neoplasms. Oncogene. https://doi.org/10.1038/s41388-020-1368-3

Elf, S., Abdelfattah, N.S., Chen, E., Perales-Patón, J., Rosen, E.A., Ko, A., Peisker, F., Florescu, N., Giannini, S., Wolach, O., Morgan, E.A., Tothova, Z., Losman, J.-A., Schneider, R.K., Al-Shahrour, F., Mullally, A., 2016. Mutant calreticulin requires both its mutant C-terminus and the thrombopoietin receptor for oncogenic transformation See supplementary information for: Reagents, cell culture, RNA sequencing and statistical analyses for RNA sequencing. HHS Public Access. Cancer Discov 6, 368-381. https://doi.org/10.1158/2159-8290.CD-15-1434.Mutant

Escolar, G., White, J.G., 1991. The platelet open canalicular system: a final common pathway. Blood Cells 17, 467-85; discussion 486-95.

George, J.N., 2000. Platelets. Lancet 355, 1531-1539. https://doi.org/10.1016/S01406736(00)02175-9

Gomez Limia, C.E., Devalle, S., Reis, M., Sochacki, J., Carneiro, M., Madeiro da Costa, R., D’Andrea, M., Padilha, T., Zalcberg, I.R., Solza, C., Daumas, A., Rehen, S., Monte-Mór, B., Bonamino, M.H., 2017. Generation and characterization of a human induced pluripotent stem (iPS) cell line derived from an acute myeloid leukemia patient evolving 
from primary myelofibrosis carrying the CALR 52 bp deletion and the ASXL1 p.R693X mutation. Stem Cell Res. 24, 16-20. https://doi.org/10.1016/j.scr.2017.08.006

Gomez Limia, C.E., Devalle, S., Reis, M., Sochacki, J., Madeiro da Costa, R., D’Andrea, M., Padilha, T., Zalcberg, I.R., Solza, C., Daumas, A., Rehen, S., Bonamino, M.H., MonteMór, B., 2018. Characterization of a human induced Pluripotent Stem (iPS) cell line (INCABRi002-A) derived from a primary myelofibrosis patient harboring the 5-bp insertion in CALR and the p.W146X mutation in TP53. Stem Cell Res. 33, 130-134. https://doi.org/10.1016/j.scr.2018.09.012

Han, L., Czech, J., Maurer, A., Brümmendorf, T.H., Chatain, N., Koschmieder, S., 2018. Mutant NRAS Q61K is responsible for MAPK pathway activation in the MARIMO cell line and renders these cells independent of the CALR-MPL-JAK2-STAT5 pathway. Leukemia 32, 2087-2090. https://doi.org/10.1038/s41375-018-0234-6

Han, L., Schubert, C., Köhler, J., Schemionek, M., Isfort, S., Brümmendorf, T.H., Koschmieder, S., Chatain, N., 2016. Calreticulin-mutant proteins induce megakaryocytic signaling to transform hematopoietic cells and undergo accelerated degradation and Golgi-mediated secretion. J. Hematol. Oncol. 9, 45. https://doi.org/10.1186/s13045-0160275-0

Irigoyen, M., García-Ruiz, J.C., Berra, E., 2017. The hypoxia signalling pathway in haematological malignancies. Oncotarget 8, 36832-36844. https://doi.org/10.18632/oncotarget.15981

Kirschner, M., Maurer, A., Wlodarski, M.W., Ventura Ferreira, M.S., Bouillon, A.-S., Halfmeyer, I., Blau, W., Kreuter, M., Rosewich, M., Corbacioglu, S., Beck, J., Schwarz, M., Bittenbring, J., Radsak, M.P., Wilk, C.M., Koschmieder, S., Begemann, M., Kurth, I., Schemionek, M., Brümmendorf, T.H., Beier, F., 2018. Recurrent somatic mutations are rare in patients with cryptic dyskeratosis congenita. Leukemia.

https://doi.org/10.1038/s41375-018-0125-x

Klampfl, T., Gisslinger, H., Harutyunyan, A.S., Nivarthi, H., Rumi, E., Milosevic, J.D., Them, N.C.C., Berg, T., Gisslinger, B., Pietra, D., Chen, D., Vladimer, G.I., Bagienski, K., Milanesi, C., Casetti, I.C., Sant'Antonio, E., Ferretti, V., Elena, C., Schischlik, F., Cleary, C., Six, M., Schalling, M., Schönegger, A., Bock, C., Malcovati, L., Pascutto, C., SupertiFurga, G., Cazzola, M., Kralovics, R., 2013. Somatic mutations of calreticulin in myeloproliferative neoplasms. N. Engl. J. Med. 369, 2379-90.

https://doi.org/10.1056/NEJMoa1311347

Kollmann, K., Nangalia, J., Warsch, W., Quentmeier, H., Bench, A., Boyd, E., Scott, M., 
Drexler, H.G., Green, A.R., 2015. MARIMO cells harbor a CALR mutation but are not dependent on JAK2/STAT5 signaling. Leukemia 29, 494-7.

https://doi.org/10.1038/leu.2014.285

Kollmann, K., Warsch, W., Gonzalez-Arias, C., Nice, F.L., Avezov, E., Milburn, J., Li, J., Dimitropoulou, D., Biddie, S., Wang, M., Poynton, E., Colzani, M., Tijssen, M.R., Anand, S., McDermott, U., Huntly, B., Green, T., 2017. A novel signalling screen demonstrates that CALR mutations activate essential MAPK signalling and facilitate megakaryocyte differentiation. Leukemia 31, 934-944. https://doi.org/10.1038/leu.2016.280

Kovarova, M., Koller, B., 2012. Differentiation of mast cells from embryonic stem cells. Curr. Protoc. Immunol. https://doi.org/10.1002/0471142735.im22f10s97

Law, C.W., Chen, Y., Shi, W., Smyth, G.K., 2014. Voom: Precision weights unlock linear model analysis tools for RNA-seq read counts. Genome Biol. 15, 1-17. https://doi.org/10.1186/gb-2014-15-2-r29

Li, J., Prins, D., Park, H.J., Grinfeld, J., Gonzalez-Arias, C., Loughran, S., Dovey, O.M., Klampfl, T., Bennett, C., Hamilton, T.L., Pask, D.C., Sneade, R., Williams, M., Aungier, J., Ghevaert, C., Vassiliou, G.S., Kent, D.G., Green, A.R., 2018. Mutant calreticulin knockin mice develop thrombocytosis andmyelofibrosis without a stemcell self-renewal advantage. Blood 131, 649-661. https://doi.org/10.1182/blood-2017-09-806356

Liu, Y., Wang, Y., Gao, Y., Forbes, J.A., Qayyum, R., Becker, L., Cheng, L., Wang, Z.Z., 2015. Efficient generation of megakaryocytes from human induced pluripotent stem cells using food and drug administration-approved pharmacological reagents. Stem Cells Transl. Med. 4, 309-19. https://doi.org/10.5966/sctm.2014-0183

Malherbe, J.A.J., Fuller, K.A., Arshad, A., Nangalia, J., Romeo, G., Hall, S.L., Meehan, K.S., Guo, B., Howman, R., Erber, W.N., 2016. Megakaryocytic hyperplasia in myeloproliferative neoplasms is driven by disordered proliferative, apoptotic and epigenetic mechanisms. J. Clin. Pathol. 69, 155-163. https://doi.org/10.1136/jclinpath2015-203177

Marinaccio, C., Suraneni, P., Celik, H., Volk, A., Wen, Q.J., Ling, T., Bulic, M., Lasho, T., Koche, R.P., Famulare, C.A., Farnoud, N., Stein, B., Schieber, M., Gurbuxani, S., Root, D.E., Younger, S.T., Hoffman, R., Gangat, N., Ntziachristos, P., Chandel, N.S., Levine, R.L., Rampal, R.K., Challen, G.A., Tefferi, A., Crispino, J.D., 2021. LKB1/ STK11 Is a Tumor Suppressor in the Progression of Myeloproliferative Neoplasms . Cancer Discov. 11, 1398-1410. https://doi.org/10.1158/2159-8290.cd-20-1353

Marty, C., Pecquet, C., Nivarthi, H., El-Khoury, M., Chachoua, I., Tulliez, M., Villeval, J.-L., 
Raslova, H., Kralovics, R., Constantinescu, S.N., Plo, I., Vainchenker, W., 2016.

Calreticulin mutants in mice induce an MPL-dependent thrombocytosis with frequent progression to myelofibrosis. Blood 127, 1317-24. https://doi.org/10.1182/blood-2015$11-679571$

Masubuchi, N., Araki, M., Yang, Y., Hayashi, E., Imai, M., Edahiro, Y., Hironaka, Y., Mizukami, Y., Kihara, Y., Takei, H., Nudejima, M., Koike, M., Ohsaka, A., Komatsu, N., 2020. Mutant calreticulin interacts with MPL in the secretion pathway for activation on the cell surface. Leukemia 34, 499-509. https://doi.org/10.1038/s41375-019-0564-z

Nangalia, J., Massie, C.E., Baxter, E.J., Nice, F.L., Gundem, G., Wedge, D.C., Avezov, E., Li, J., Kollmann, K., Kent, D.G., Aziz, A., Godfrey, A.L., Hinton, J., Martincorena, I., Van Loo, P., Jones, A. V, Guglielmelli, P., Tarpey, P., Harding, H.P., Fitzpatrick, J.D., Goudie, C.T., Ortmann, C.A., Loughran, S.J., Raine, K., Jones, D.R., Butler, A.P., Teague, J.W., O’Meara, S., McLaren, S., Bianchi, M., Silber, Y., Dimitropoulou, D., Bloxham, D., Mudie, L., Maddison, M., Robinson, B., Keohane, C., Maclean, C., Hill, K., Orchard, K., Tauro, S., Du, M.-Q., Greaves, M., Bowen, D., Huntly, B.J.P., Harrison, C.N., Cross, N.C.P., Ron, D., Vannucchi, A.M., Papaemmanuil, E., Campbell, P.J., Green, A.R., 2013. Somatic CALR mutations in myeloproliferative neoplasms with nonmutated JAK2. N. Engl. J. Med. 369, 2391-2405.

https://doi.org/10.1056/NEJMoa1312542

Nauseef, W.M., McCormick, S.J., Clark, R.A., 1995. Calreticulin functions as a molecular chaperone in the biosynthesis of myeloperoxidase. J. Biol. Chem. 270, 4741-4747. https://doi.org/10.1074/jbc.270.9.4741

Noetzli, L.J., French, S.L., Machlus, K.R., 2019. New insights into the differentiation of megakaryocytes from hematopoietic progenitors. Arterioscler. Thromb. Vasc. Biol. 39, 1288-1300. https://doi.org/10.1161/ATVBAHA.119.312129

Pecquet, C., Chachoua, I., Roy, A., Balligand, T., Vertenoeil, G., Leroy, E., Albu, R.-I., Defour, J.-P., Nivarthi, H., Hug, E., Xu, E., Ould-Amer, Y., Mouton, C., Colau, D., Vertommen, D., Shwe, M.M., Marty, C., Plo, I., Vainchenker, W., Kralovics, R., Constantinescu, S.N., 2019. Calreticulin mutants as oncogenic rogue chaperones for TpoR and traffic-defective pathogenic TpoR mutants. Blood 133, 2669-2681. https://doi.org/10.1182/blood-2018-09-874578

Psaila, B., Wang, G., Rodriguez-Meira, A., Li, R., Heuston, E.F., Murphy, L., Yee, D., Hitchcock, I.S., Sousos, N., O'Sullivan, J., Anderson, S., Senis, Y.A., Weinberg, O.K., Calicchio, M.L., Iskander, D., Royston, D., Milojkovic, D., Roberts, I., Bodine, D.M., Thongjuea, S., Mead, A.J., 2020. Single-Cell Analyses Reveal Megakaryocyte-Biased 
Hematopoiesis in Myelofibrosis and Identify Mutant Clone-Specific Targets. Mol. Cell 78, 477-492.e8. https://doi.org/10.1016/j.molcel.2020.04.008

Robinson, M.D., Oshlack, A., 2010. A scaling normalization method for differential expression analysis of RNA-seq data. Genome Biol. 11, R25. https://doi.org/10.1186/gb2010-11-3-r25

Ru, Y.X., Zhao, S.X., Dong, S.X., Yang, Y.Q., Eyden, B., 2015. On the Maturation of Megakaryocytes: A Review with Original Observations on Human in Vivo Cells Emphasizing Morphology and Ultrastructure. Ultrastruct. Pathol. 39, 79-87. https://doi.org/10.3109/01913123.2014.980482

Schneider, C. a, Rasband, W.S., Eliceiri, K.W., 2012. NIH Image to ImageJ: 25 years of image analysis. Nat. Methods 9, 671-5. https://doi.org/10.1038/nmeth.2089

Schubert, M., Klinger, B., Klünemann, M., Sieber, A., Uhlitz, F., Sauer, S., Garnett, M.J., Blüthgen, N., Saez-Rodriguez, J., 2018. Perturbation-response genes reveal signaling footprints in cancer gene expression. Nat. Commun. 9, 20. https://doi.org/10.1038/s41467-017-02391-6

Secardin, L., Gomez Limia, C., da Silva-Benedito, S., Lordier, L., El-Khoury, M., Marty, C., lanotto, J.-C., Raslova, H., Constantinescu, S.N., Bonamino, M.H., Vainchenker, W., Monte-Mor, B., Di Stefano, A., Plo, I., 2021. Induced Pluripotent Stem Cells Enable Disease Modeling and Drug Screening in Calreticulin del52 and ins5 Myeloproliferative Neoplasms. HemaSphere 5, e593. https://doi.org/10.1097/HS9.0000000000000593

Semenza, G.L., 2009. Regulation of Oxygen Homeostasis by Hypoxia-Inducible Factor 1. Physiology 24, 97-106. https://doi.org/10.1152/physiol.00045.2008

Shide, K., Kameda, T., Kamiunten, A., Oji, A., Ozono, Y., Sekine, M., Honda, A., Kitanaka, A., Akizuki, K., Tahira, Y., Nakamura, K., Hidaka, T., Kubuki, Y., Abe, H., Miike, T., Iwakiri, H., Tahara, Y., Sueta, M., Hasuike, S., Yamamoto, S., Nagata, K., 2019. Mice with Calr mutations homologous to human CALR mutations only exhibit mild thrombocytosis. Blood Cancer J. https://doi.org/10.1038/s41408-019-0202-z

Sim, X., Jarocha, D., Hayes, V., Hanby, H.A., Marks, M.S., Camire, R.M., French, D.L., Poncz, M., Gadue, P., 2017. Identifying and enriching platelet-producing human Stem cell-derived megakaryocytes using factor V uptake. Blood 130, 192-204. https://doi.org/10.1182/blood-2017-01-761049

Stengel, A., Jeromin, S., Haferlach, T., Meggendorfer, M., Kern, W., Haferlach, C., 2019. Detection and characterization of homozygosity of mutated CALR by copy neutral loss 
of heterozygosity in myeloproliferative neoplasms among cases with high CALR mutation loads or with progressive disease. Haematologica 104, e187-e190. https://doi.org/10.3324/haematol.2018.202952

Sugimura, R., Jha, D.K., Han, A., Soria-Valles, C., Da Rocha, E.L., Lu, Y.F., Goettel, J.A., Serrao, E., Rowe, R.G., Malleshaiah, M., Wong, I., Sousa, P., Zhu, T.N., Ditadi, A., Keller, G., Engelman, A.N., Snapper, S.B., Doulatov, S., Daley, G.Q., 2017.

Haematopoietic stem and progenitor cells from human pluripotent stem cells. Nature 545, 432-438. https://doi.org/10.1038/nature22370

Swerdlow, S.H., Campo, E., Harris, N.L., Jaffe, E.S., Pileri, S., Stein, H., Thiele, J., 2008. WHO Classification of Tumours of Haematopoietic and Lymphoid Tissues, 4th edn. ed.

Takahashi, K., Tanabe, K., Ohnuki, M., Narita, M., Ichisaka, T., Tomoda, K., Yamanaka, S., 2007. Induction of pluripotent stem cells from adult human fibroblasts by defined factors. Cell 131, 861-72. https://doi.org/10.1016/j.cell.2007.11.019

Takei, H., Edahiro, Y., Mano, S., Masubuchi, N., Mizukami, Y., Imai, M., Morishita, S., Misawa, K., Ochiai, T., Tsuneda, S., Endo, H., Nakamura, S., Eto, K., Ohsaka, A., Araki, M., Komatsu, N., 2018. Skewed megakaryopoiesis in human induced pluripotent stem cell-derived haematopoietic progenitor cells harbouring calreticulin mutations. $\mathrm{Br}$. J. Haematol. 181, 791-802. https://doi.org/10.1111/bjh.15266

Theocharides, A.P.A., Lundberg, P., Lakkaraju, A.K.K., Lysenko, V., Myburgh, R., Aguzzi, A., Skoda, R.C., Manz, M.G., 2016. Homozygous calreticulin mutations in patients with myelofibrosis lead to acquired myeloperoxidase deficiency. Blood 127, 3253-9. https://doi.org/10.1182/blood-2016-02-696310

Thomlinson, R.H., Gray, L.H., 1955. The Histological Structure of Some Human Lung Cancers and the Possible Implications for Radiotherapy. Br. J. Cancer 9, 539-549. https://doi.org/10.1038/bjc. 1955.55

Tijssen, M.R., Ghevaert, C., 2013. Transcription factors in late megakaryopoiesis and related platelet disorders. J. Thromb. Haemost. 11, 593-604. https://doi.org/10.1111/jth.12131

Vivoli, E., Di Maira, G., Marra, F., 2016. Liver Fibrosis and Leptin. Curr. Pathobiol. Rep. 4, 69-76. https://doi.org/10.1007/s40139-016-0103-9

Wang, W., Wang, T., Kotini, A.G., lancu-Rubin, C., Hoffman, R., Papapetrou, E.P., 2018. Modeling Calreticulin-Mutant Myeloproliferative Neoplasms with Isogenic Induced Pluripotent Stem Cells. Blood 132, 4319-4319. https://doi.org/10.1182/blood-2018-99111512 
Zingariello, M., Rosti, V., Vannucchi, A.M., Guglielmelli, P., Mazzarini, M., Barosi, G., Genova, M.L., Migliaccio, A.R., 2020. Shared and Distinctive Ultrastructural Abnormalities Expressed by Megakaryocytes in Bone Marrow and Spleen From Patients With Myelofibrosis. Front. Oncol. 10, 1-12.

https://doi.org/10.3389/fonc.2020.584541 


\section{Figure Legends}

Figure 1. MPN-related iPS cell clones exhibit pluripotency state and harbor CALR mutations

(A) Pluripotency assessment of indicated iPS cell clones by immunofluorescence. Merge represents overlay of DAPI (blue), OCT3/4 (green) and TRA-1-60 (red). Scale bar, $100 \mu \mathrm{m}$. (B) CALR mRNA expression of indicated iPS cell clones was assessed by CALR mutant allelespecific RT-qPCR. Gene expression is depicted as percentage to GAPDH. Mean value \pm SD of representative clones with indicated CALR genotypes. (C) Mutant CALR expression (red) was confirmed by immunofluorescence staining with a CALR mutant specific antibody. Unmutated CALR iPS cells served as negative control. Nuclei were stained with DAPI (blue). Scale bars, $100 \mu \mathrm{m}$. (D) Representative Western blot analysis of CALR WT and CALR mutant (Mut) protein in iPS cell clones after CRISPR repair of homozygous CALRins5 and del52 mutations. CALR WT protein was assessed on the same membrane as CALR mutant without (w/o) stripping of the CALR Mut antibody explaining residual CALR mutant bands. GAPDH was used as loading control.

Figure 2. Restoration of MPO activity in iPS cells with repaired CALRins5 or del52 mutation

(A) Schematic representation of a "EB-based" differentiation protocol of iPS cells towards hematopoietic progenitors. Representative images of the differentiation procedure from EB formation to HSPC production of iPS cells are shown. Typical morphology of iPS cell colonies on day 0 , embryoid bodies on day 5 , mesoderm commitment and differentiated hemogenic endothelial layers on day 8, and HSPC production on day 25. Scale bars white and black, 1000 $\mu \mathrm{m}$ and $400 \mu \mathrm{m}$, respectively. (B) Flow cytometry gating strategy to identify cyMPO+ neutrophils on day 15 of "EB-based" differentiation. Exemplarily shown for iPS cell-derived hematopoietic cells carrying heterozygous (orange) or homozygous (red) CALR mutation or unmutated CALR (green). Numbers represent frequencies of CD15+cyMPO+ populations in percent of living single cells. (C) Flow cytometry data for cell surface CD15 and intracellular MPO expression of iPS-cell derived HSPCs on day 15 of "EB-based" differentiation. Data of three independent experiments for each $C A L R$ genotype are shown. ${ }^{*} p<0.05,{ }^{* * *} p<0.001$. (D) Relative MPO mRNA expression was confirmed by RT-qPCR. Gene expression is depicted as percentage to MT-ATP6. Experiments were performed in triplicates for each CALR genotype. Mean value $\pm \mathrm{SD}$ of representative clones carrying indicated CALR genotypes are shown. ${ }^{* *} p<0.01$. (E) MPO functional activity was assessed by cytochemical staining. Representative 
images of hematopoietic cells harboring indicated CALR genotypes are shown. The intensity of the black brown dye indicates the peroxidase activity. Scale bars, $50 \mu \mathrm{m}$.

\section{Figure 3: Hematopoietic differentiation potential of iPS cell-derived CD34+ cells}

(A) Schematic representation of a "spin-EB" protocol to differentiate iPS cells towards HSCs and MKs adapted from Liu et al. (Liu et al., 2015) .Cell culture medium was continuously supplemented with cytokines as indicated. On day 14, suspension cells were harvested for further analysis. Representative images of cell culture on indicated days of differentiation are shown. Scale bars, $50 \mu \mathrm{m}$. (B) Cell number of suspension cells on day 14 of "spin-EB" differentiation calculated for harvested suspension cells/well of 96-well plate. Each data point represents an independent experiment for indicated $C A L R$ mutation and genotype. * $p>0.05$, ** $p>0.01$. (C) Percentage of CD34+CD45+ cells on day 10, day 12 and day 14 of "spin-EB“ differentiation analyzed by flow cytometry analysis. Each data point represents an independent experiment for each $C A L R$ genotype and HD control. ${ }^{*} p<0.05,{ }^{* *} p<0.01$. (D) Colony-forming unit (CFU) assay of purified iPS-cell derived CD34+ cells on day 14 of "spin-EB" differentiation. CD34+ cells were isolated by immunogenic bead selection (MACS) and 5,000 cells $/ \mathrm{ml}$ were seeded in semisolid medium supplemented with cytokines. After 12 days, colonies were characterized and counted. Results of three independent experiments for each CALR genotype and HD controls are shown. Red dash and blue dash referred to significant differences in CFU-E and CFU-M, respectively. ${ }^{*} p>0.05,{ }^{* *} p>0.01,{ }^{* * *} p>0.001$. Morphological appearance of different colony types was assessed by cytospin preparation and Diff-Quik staining. Scale bars, $50 \mu \mathrm{m}$.

\section{Figure 4. Megakaryocytic differentiation of patient-specific CALRins5 and CALRdel52 iPS cells}

(A) Percentage of CD42b+CD41+ matured MKs determined by flow cytometry on day 14 of "spin-EB" differentiation. Numbers of independent experiments performed for each CALR genotype and HD control referred to number of data points shown. ${ }^{*} p<0.05,{ }^{* *} p<0.01$, ${ }^{* * *} p<0.001$. (B) Representative morphology of MKs harvested on day 14 of "spin-EB" differentiation exemplarily shown for CALRins5 homozygous clone and repaired WT clone $\left(\mathrm{WT}_{\mathrm{cr}}\right)$ stained with Diff-Quik solutions after cytospin preparation. Typical MKs and aberrant smaller MKs are indicated with black and red arrows, respectively. Scale bars, $50 \mu \mathrm{m}$. (C) Impact of TPO on MK development analyzed by flow cytometry analysis on day 14 of "spin-EB" differentiation. Percentage of CD42b+CD41+ MKs is shown for cells treated with $(+)$ or without (-) TPO from day 11 of differentiation onwards. Numbers of independent experiments for each $C A L R$ genotype and HD control referred to number of data point shown. ${ }^{*} p<0.05,{ }^{* *} p<0.01$, ${ }^{* * *} p<0.001$. (D) Percentage of CD61+CD41+ immature MKs determined by flow cytometry analysis. Data of day 10 , day 12 and day 14 of three independent experiments are combined 
for each $C A L R$ genotype and HD controls. ${ }^{* *} p<0.01,{ }^{* *} p<0.001$. (E) CD42b+ and CD42bcells in CD61+CD41+ MKs on day 10 and day 14 of "spin-EB" differentiation analyzed by flow cytometry analysis. Statistical analysis compares number of CD42b+ cells of homozygous clones to corresponding heterozygous and $\mathrm{WT}_{\mathrm{cr}}$ clones of three independent experiments. ${ }^{* *} p<0.01$.

\section{Figure 5. Gene expression profile of iPS cell-derived MKs harboring CALR mutations}

(A) and (B) Heat map for unsupervised hierarchical linkage clustering of selected MK-related genes analyzed by RT-qPCR of CD61+ cells. CD61 + cells were isolated by immunogenic bead selection (MACS) on day 14 of "spin-EB" differentiation. Data are normalized to gene expression of purified CD34+ cells of the same experiment. Calculated z-score is shown for individual experiment of indicated CALR genotype (ins5 (A), del52 (B), red and blue, high and low expression; respectively). (C) Gene expression profile of MPL, FLI-1, NF-E2, and VWF of CD61+ MKs. Expression was normalized to CD34+ cells from the same experiment. Analysis was performed for three independent experiments performed for each CALRins 5 genotype. Statistical analysis compares the expression of homozygous and heterozygous CALR-mutated MKs to $W T_{\text {cr }}$ MK. ${ }^{*} p<0.05,{ }^{* *} p<0.01,{ }^{* * *} p<0.001$.

Figure 6. Ultrastructural morphology analysis of iPS cell-derived MKs and CALR distribution in MKs

(A) Transmission electron microscopy (TEM) images of iPS cell-derived MKs of indicated CALRins5 genotype to evaluate cell morphology. Representative images are shown. $\mathrm{N}$ : nucleus. DMS: Demarcation membrane system. *: pro-platelet protrusions. Black arrow: granules. Yellow arrow: open canalicular system. m: mitochondria. Scale bars, $2.5 \mu \mathrm{m}$ (large panels) and $500 \mathrm{~nm}$ (small panels). (B) Calculated cell area of MKs in TEM images for indicated CALRins5 genotypes. Each data point represents a single cell. (C) Mean fluorescence intensity (MFI) calculated for the side-scatter (SSC-A) of CD42b+CD41+CD61+ MKs on day 14 of "spin-EB" differentiation. Indicated data points represent independent experiments for each CALRins5 and del52 genotype. ${ }^{* *} p<0.01$. (D) iPS cell-derived MKs were fixed and stained for the endoplasmic reticulum (ER) and WT CALR or mutated (Mut) CALR after 14 days of differentiation for indicated CALRins5iPS cell clones. To identify MKs, samples were additionally stained for CD42b. Hoechst was added for nuclear staining. Scale bars, $50 \mu \mathrm{m}$. Diffuse CALR distribution, clustered localization of CALR at the cell surface, and colocalization of CALR and ER are indicated with yellow, green, and orange arrows, respectively

Figure 7. RNAseq experiments with iPS cell-derived MKs of CALRins5-mutated clones

(A) Heat map for unsupervised hierarchical clustering of RNAseq data of iPS cell-derived MKs with homozygous (Hom) or heterozygous (Het) CALRins5 mutation or unmutated $\left(\mathrm{WT}_{\mathrm{cr}}\right) \mathrm{MKs}$. 
Numbers represent independent experiments. Unsupervised clustering was performed. Cluster 1 and cluster 2 are indicated in grey and black, respectively. (B) Gene expression profile of FN1 in iPS cell-derives MKs of RNAseq analysis. Analysis was performed for three independent experiments. ${ }^{* *} p<0.01$. (C) Pathway Responsive genes (PROGENy) of multiple comparisons of Hom, Het and $\mathrm{WT}_{\mathrm{cr}}$ iPS cell-derived MKs. Upregulated and downregulated pathways were shown in red and blue, respectively. Adjusted p-values from multiple comparisons are shown in the table. Significant values (p.adjusted<0.05) are highlighted in red. (D) Gene expression profile of NDRG1 and LEP in iPS cell-derives MKs of RNAseq analysis. Analysis was performed for three independent experiments. ${ }^{*} p<0.05,{ }^{* *} p<0.01$. 
Figure 1

$A_{\text {CALR }}$ del31

del52
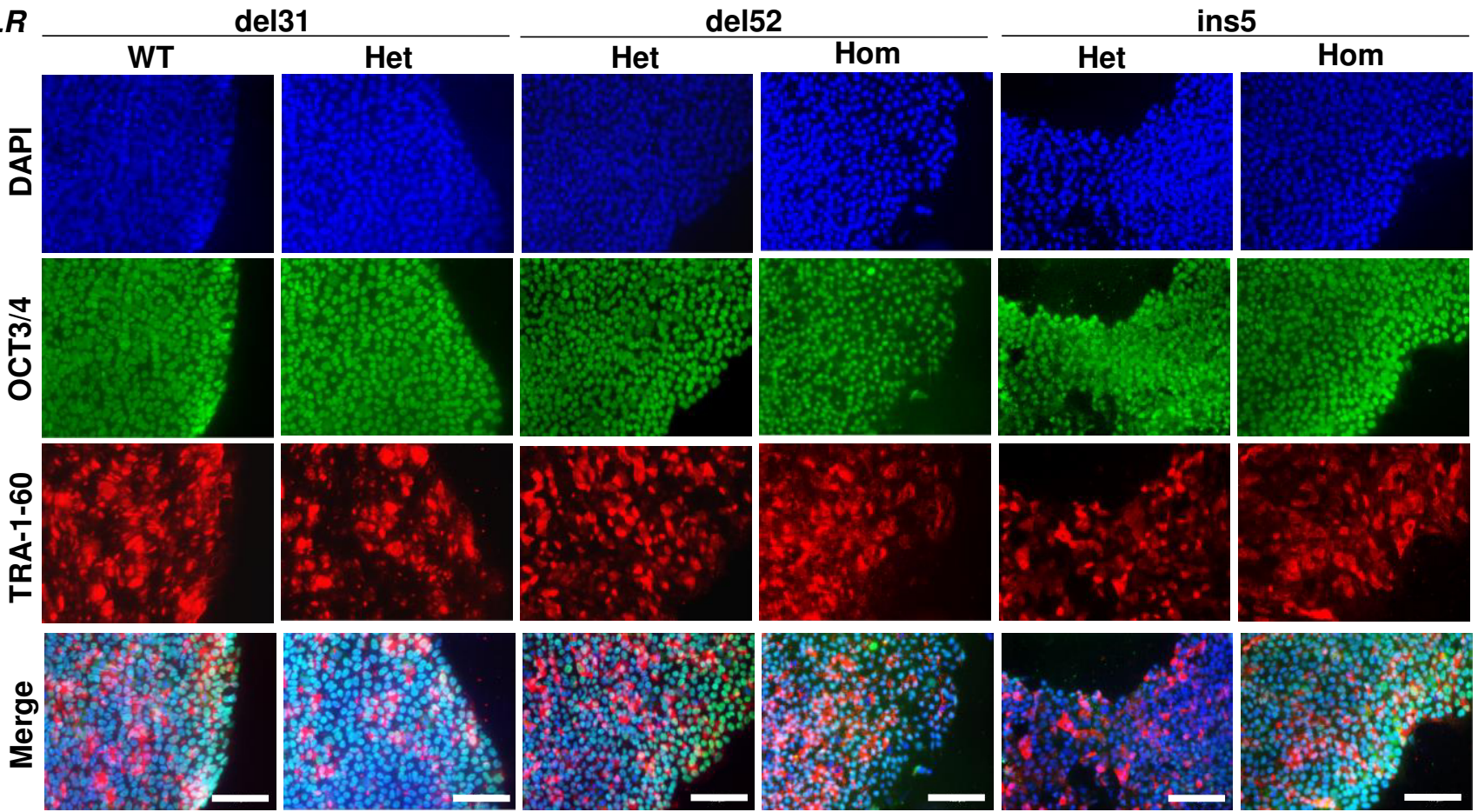

B

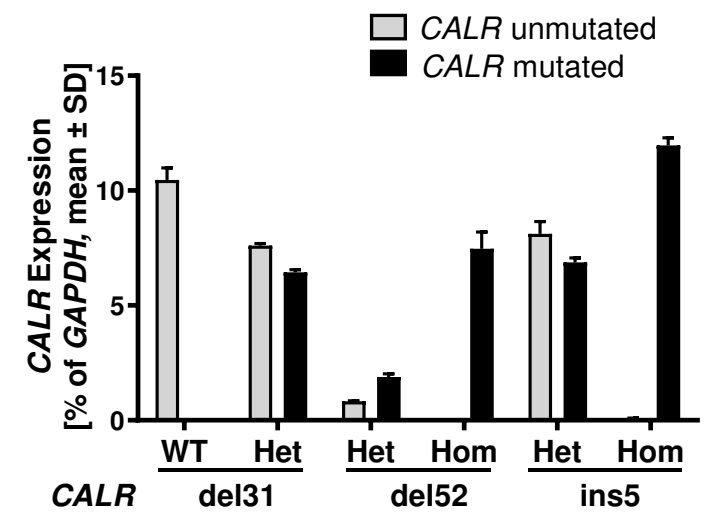

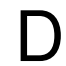

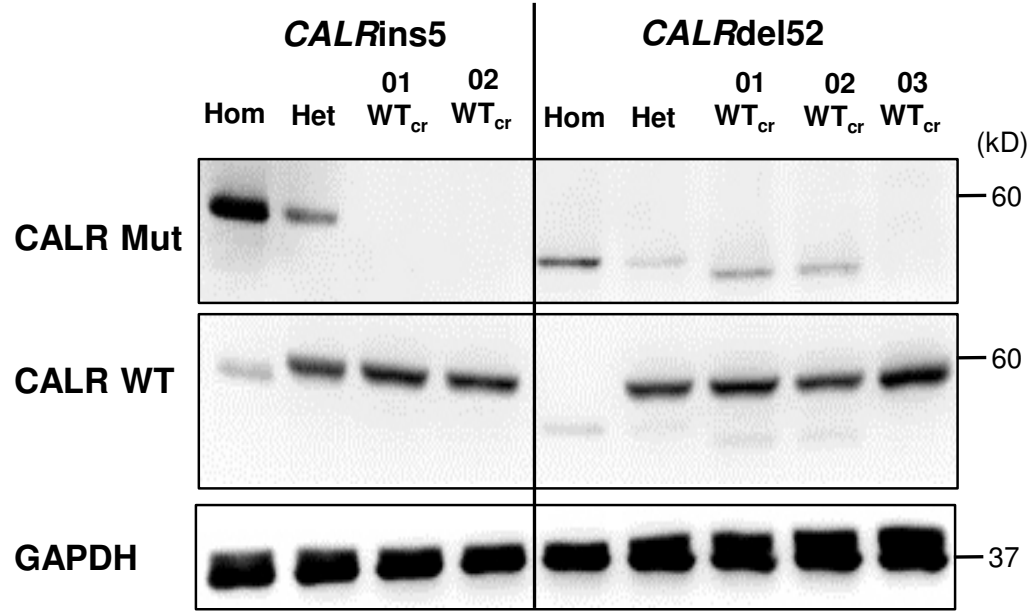

C

CALR

del31
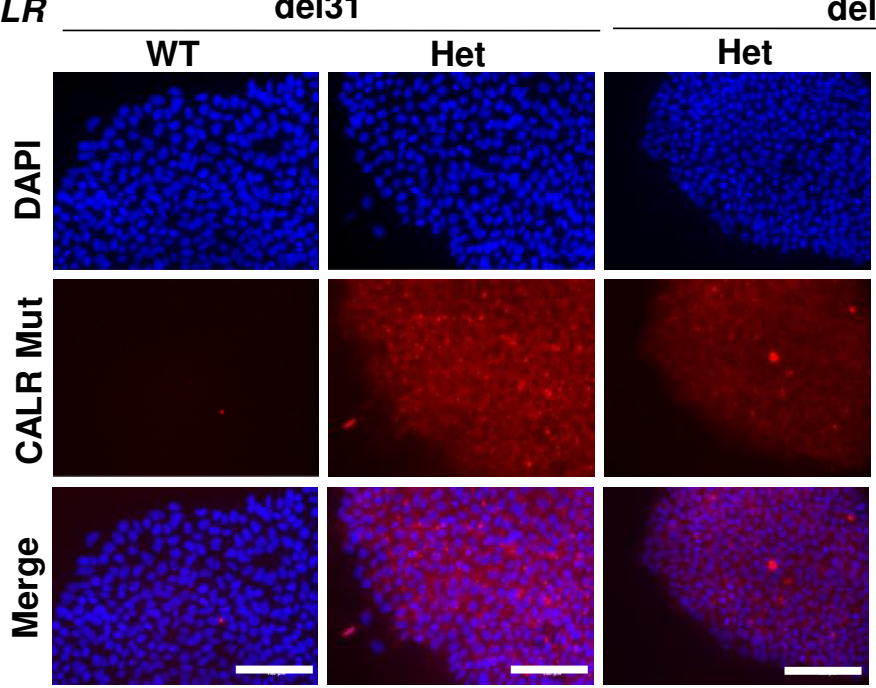

del52

Hom
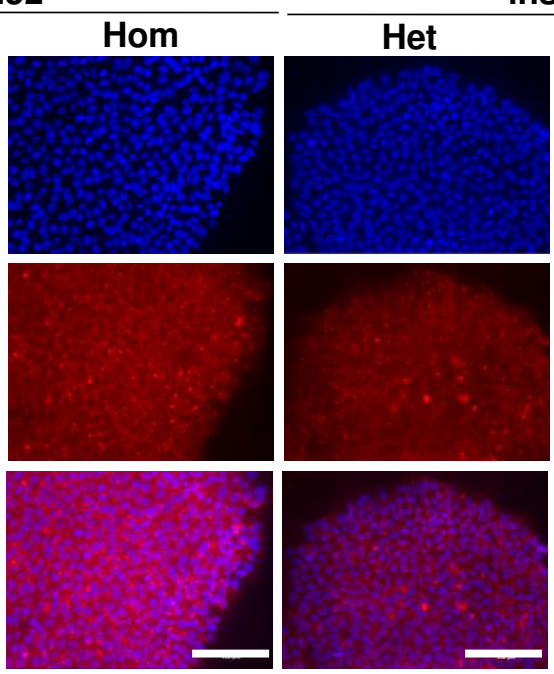

ins5

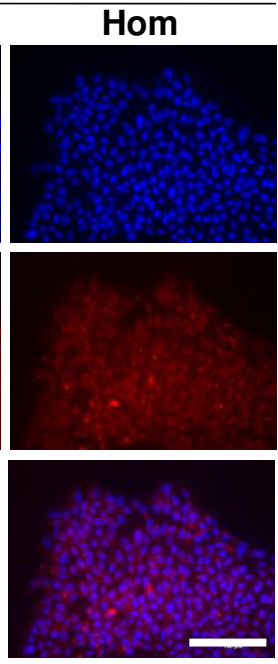


Figure 2

A

CD34+

MACS
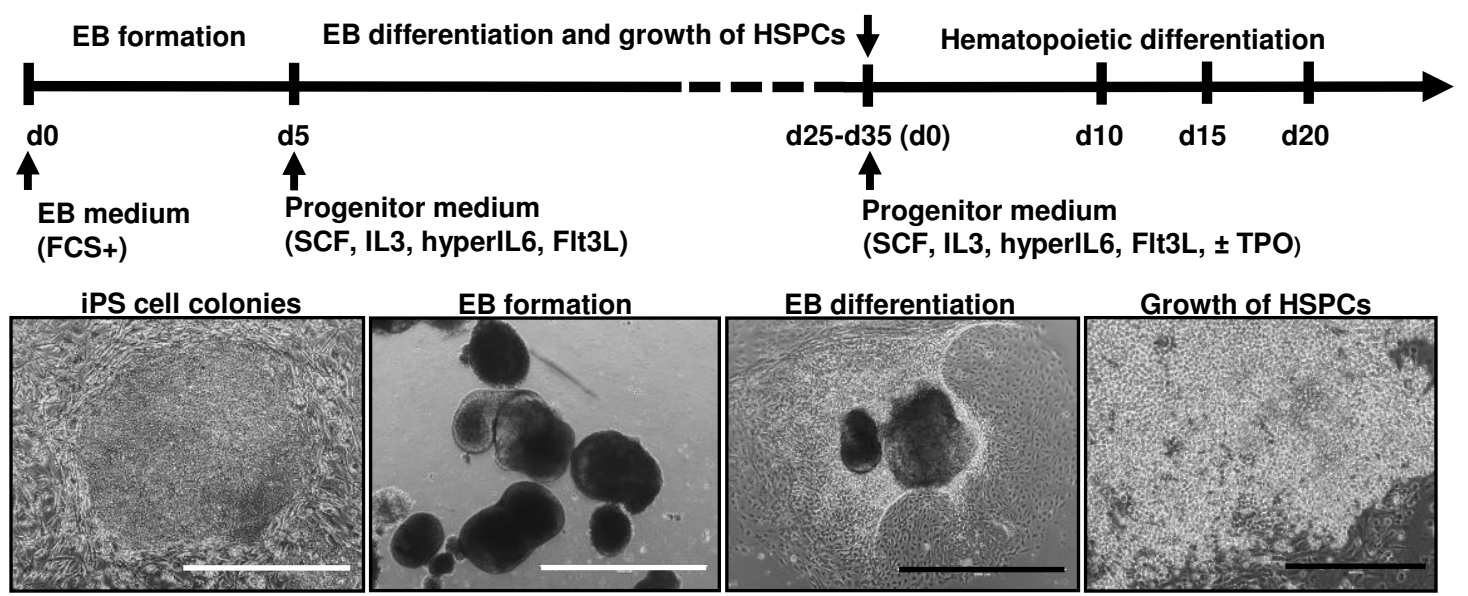

EB differentiation Growth of HSPCs

B
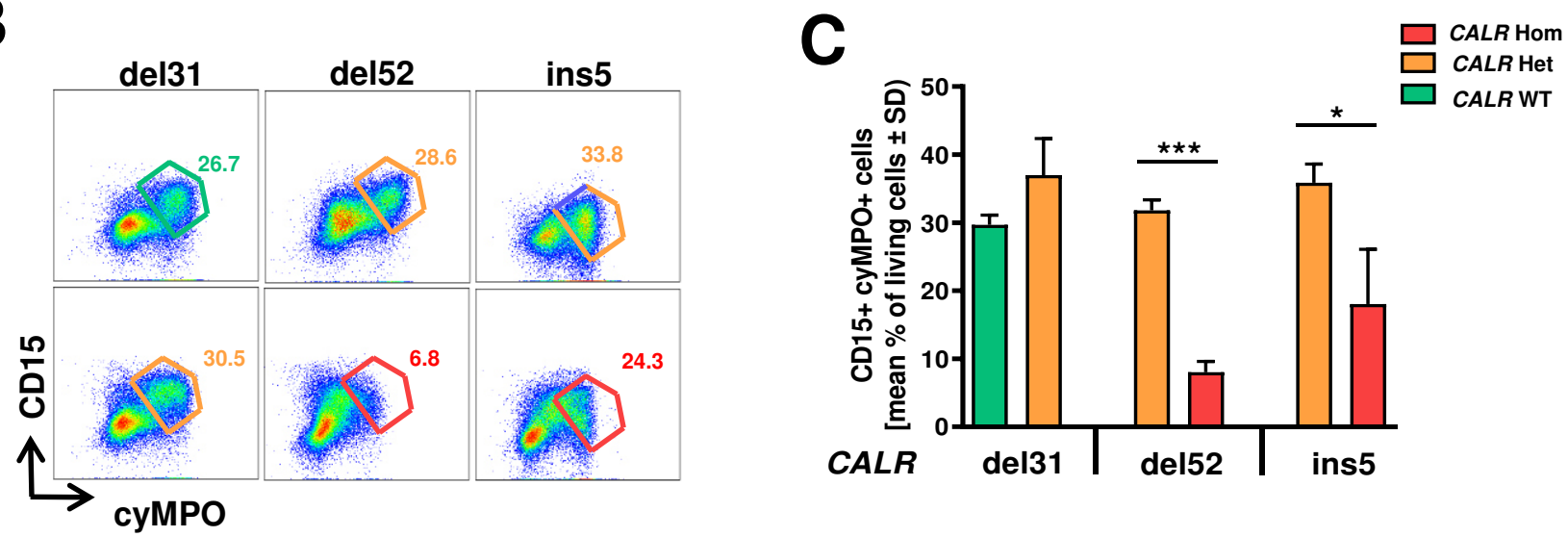

$D$
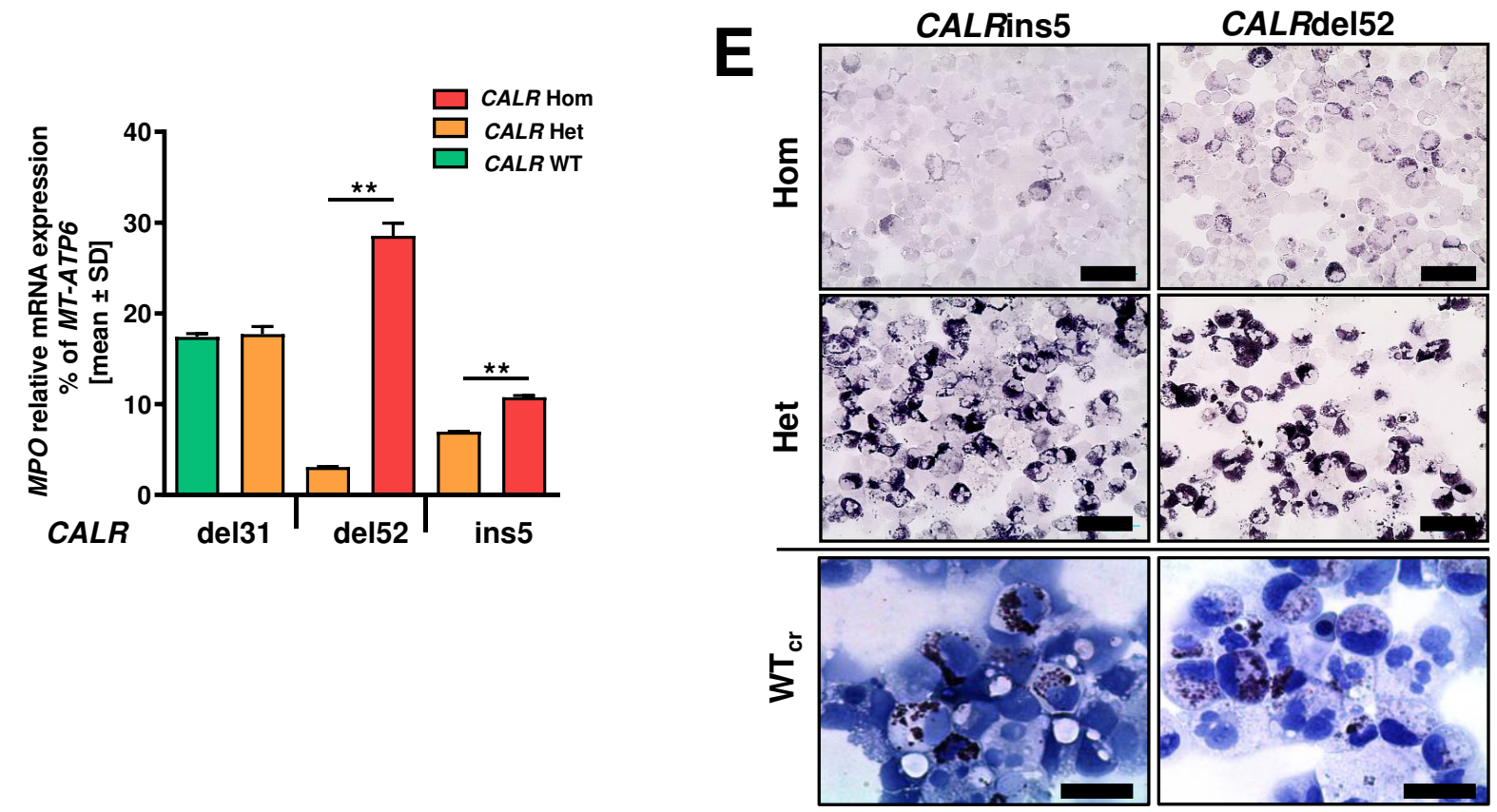
Figure 3

A
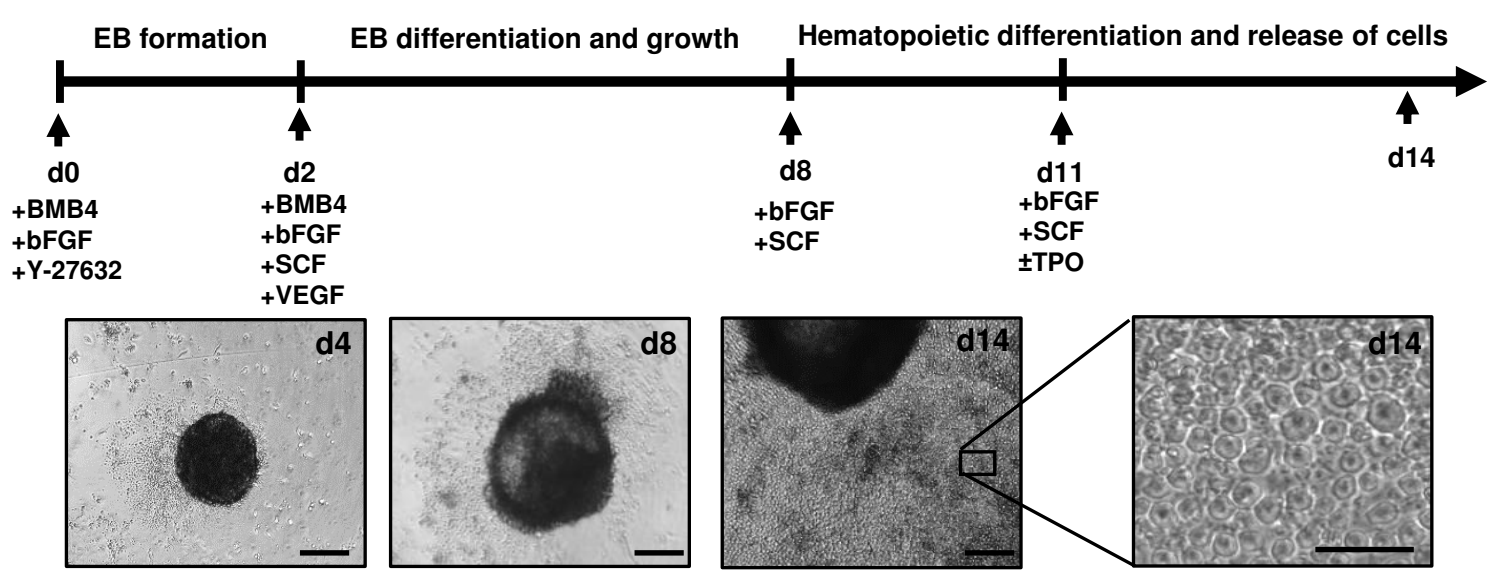

B

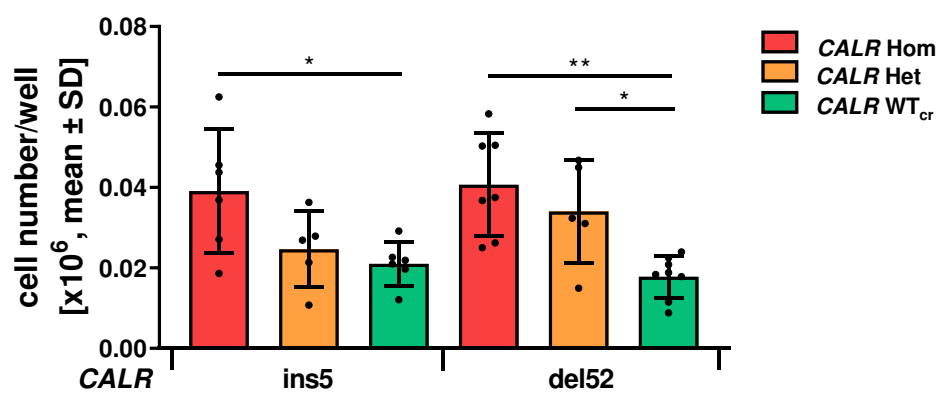

C

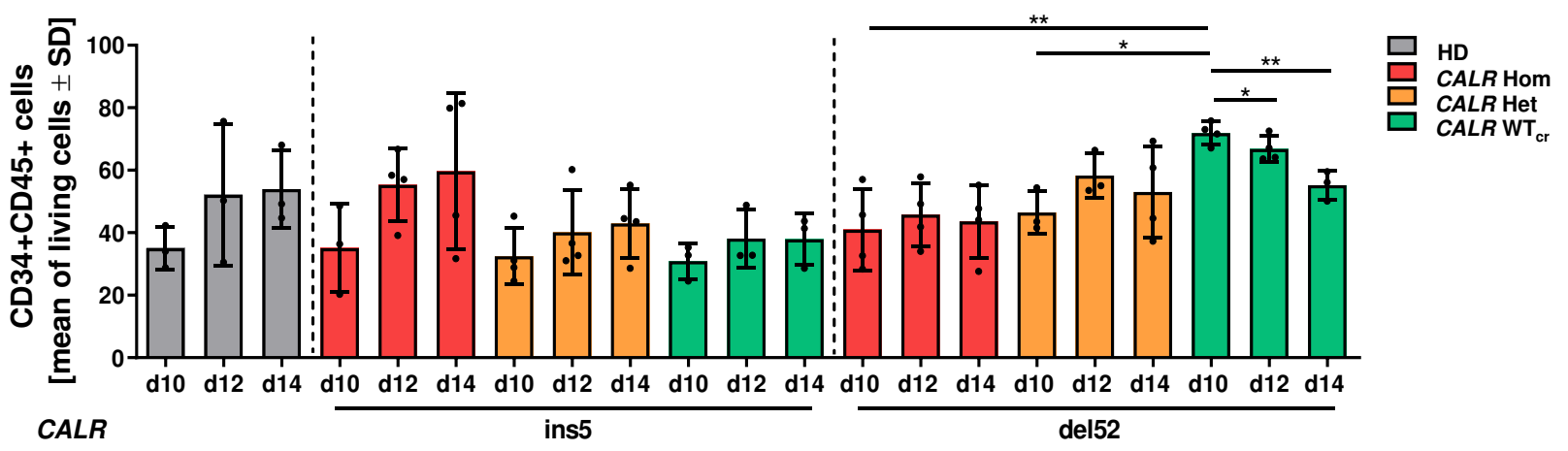

D

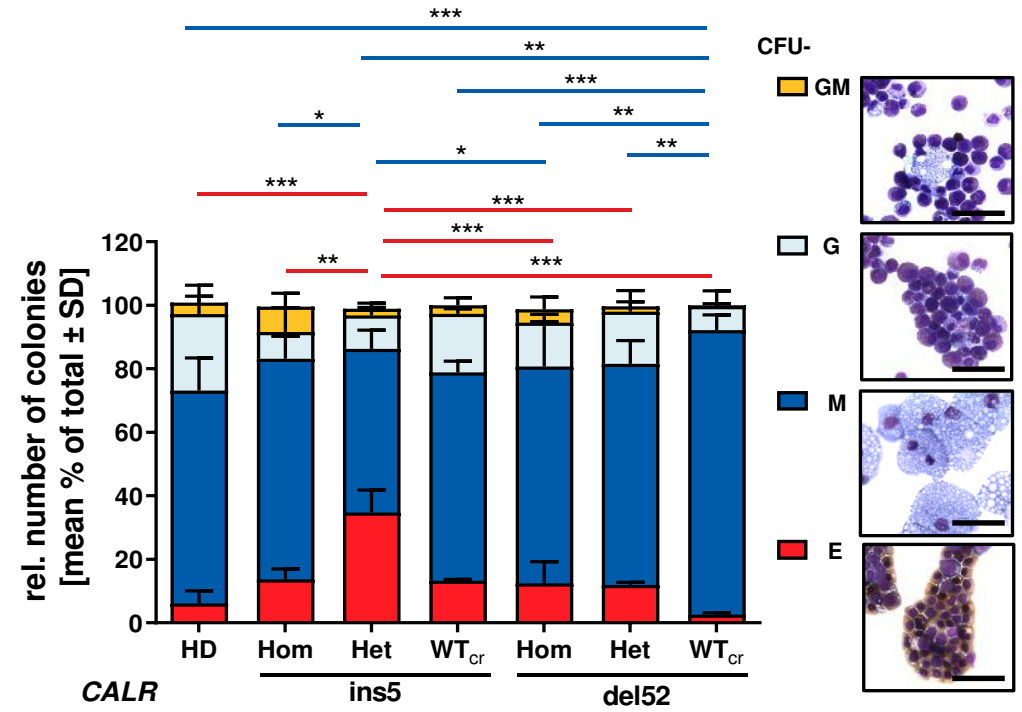


Figure 4

A
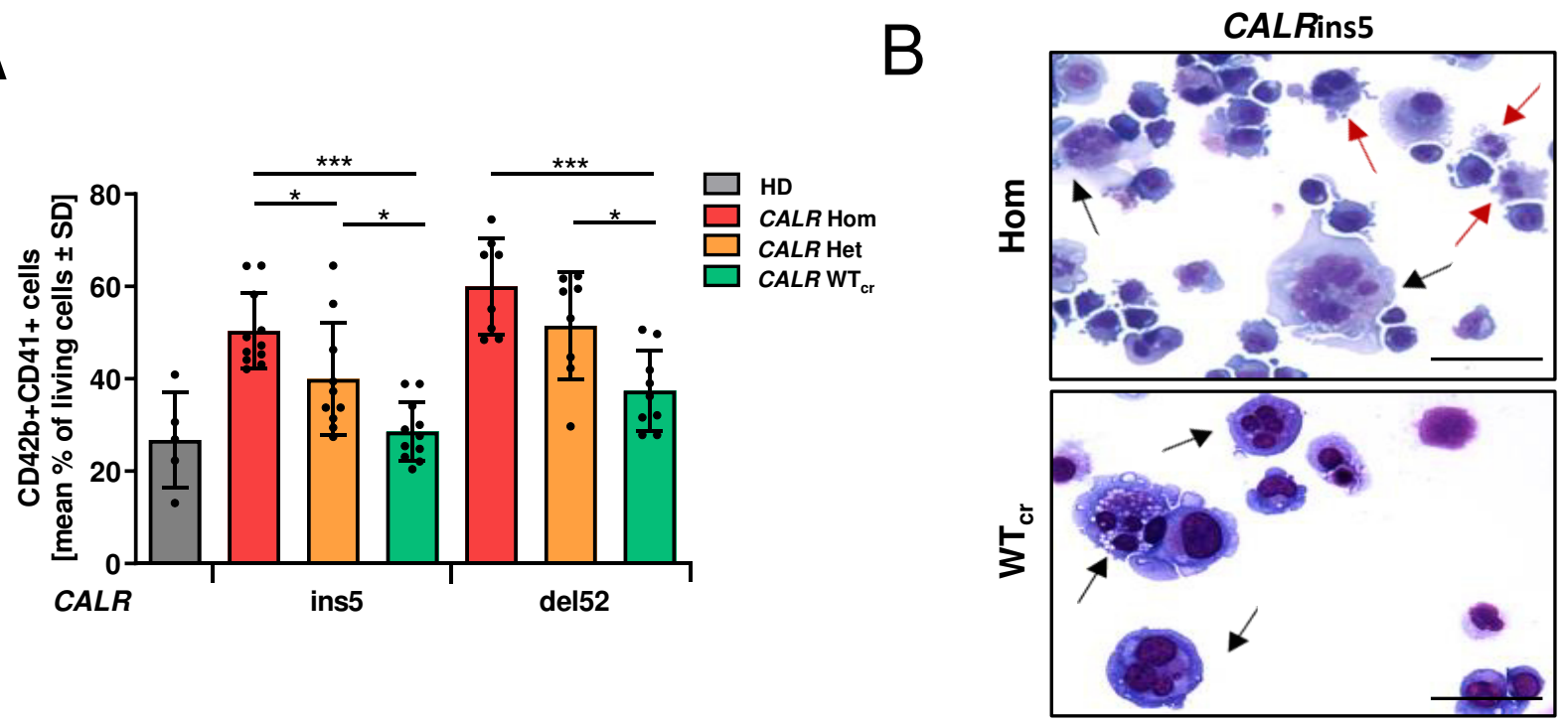

C

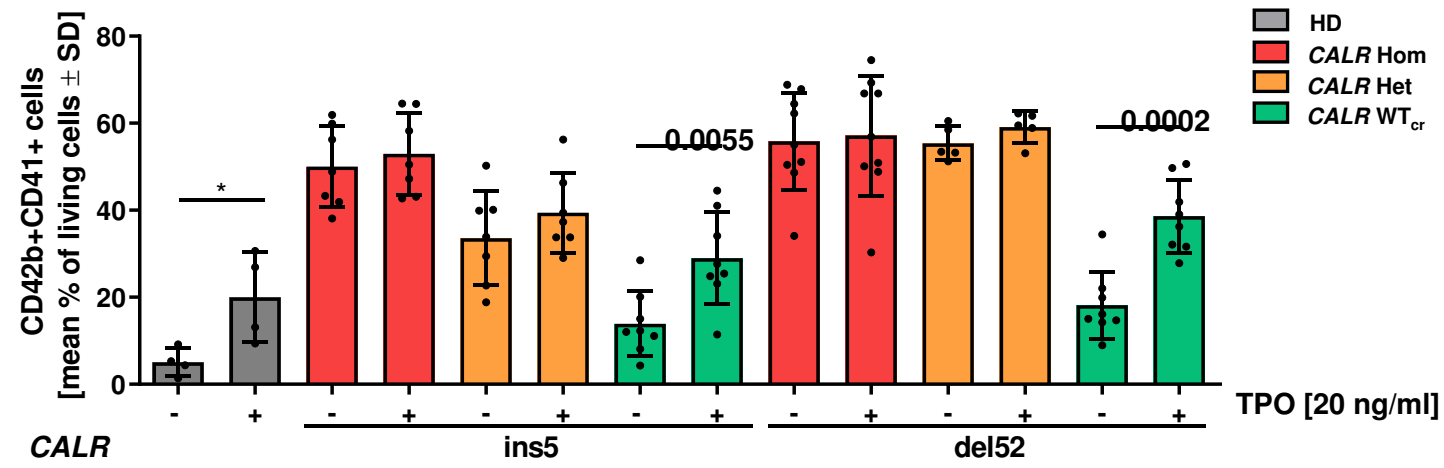

D

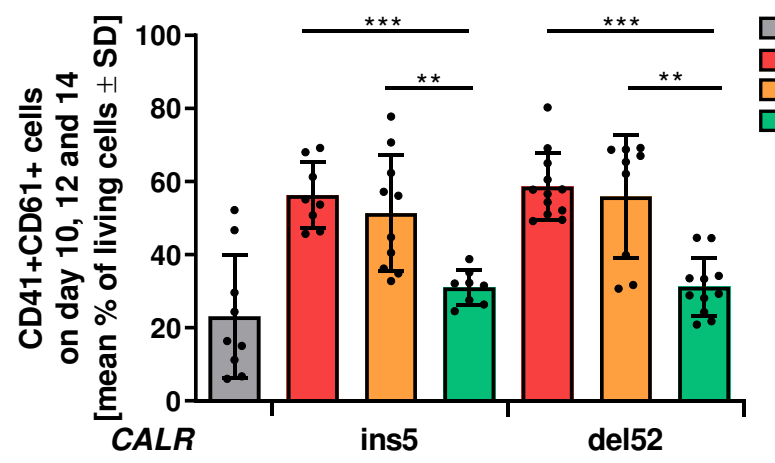

$E$
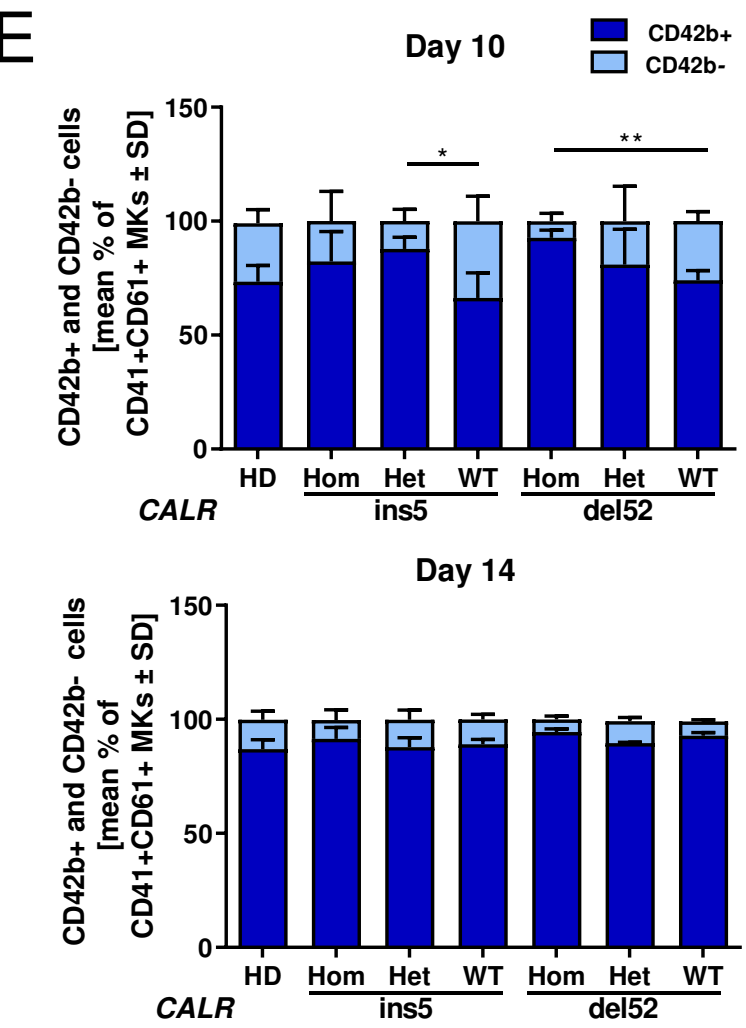
Figure 5

A

\section{CALRins5}

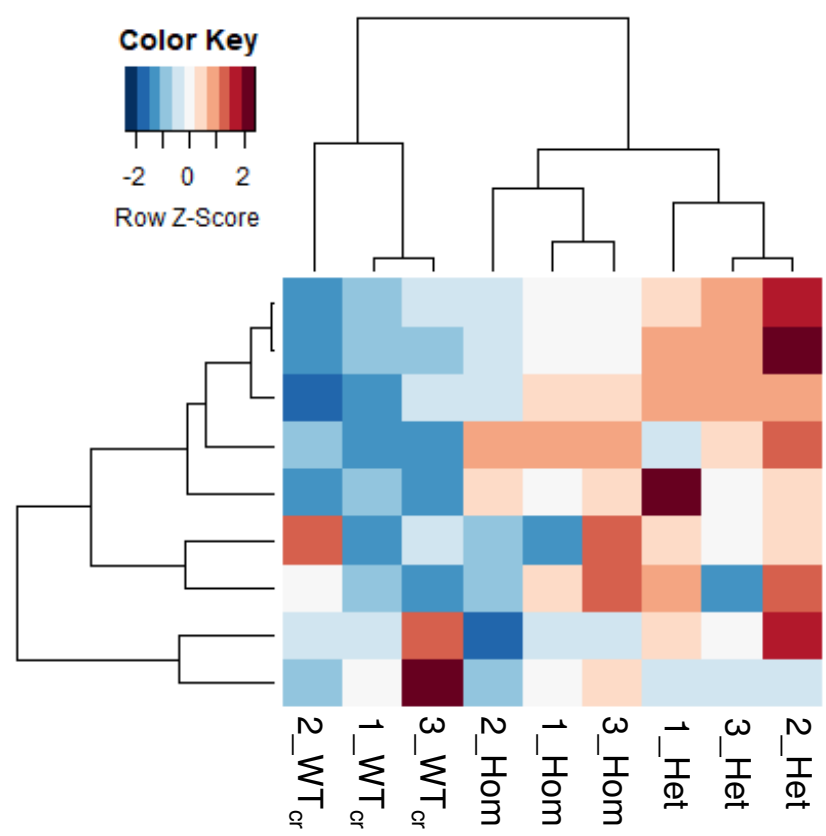

B

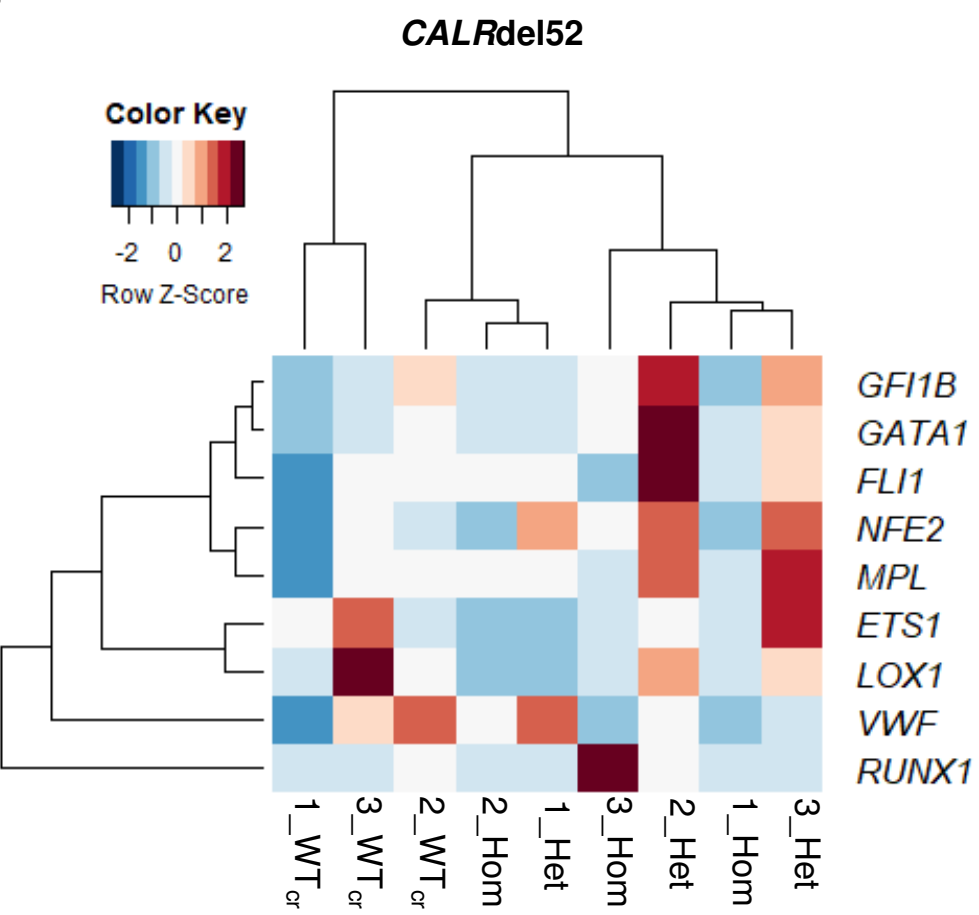

C
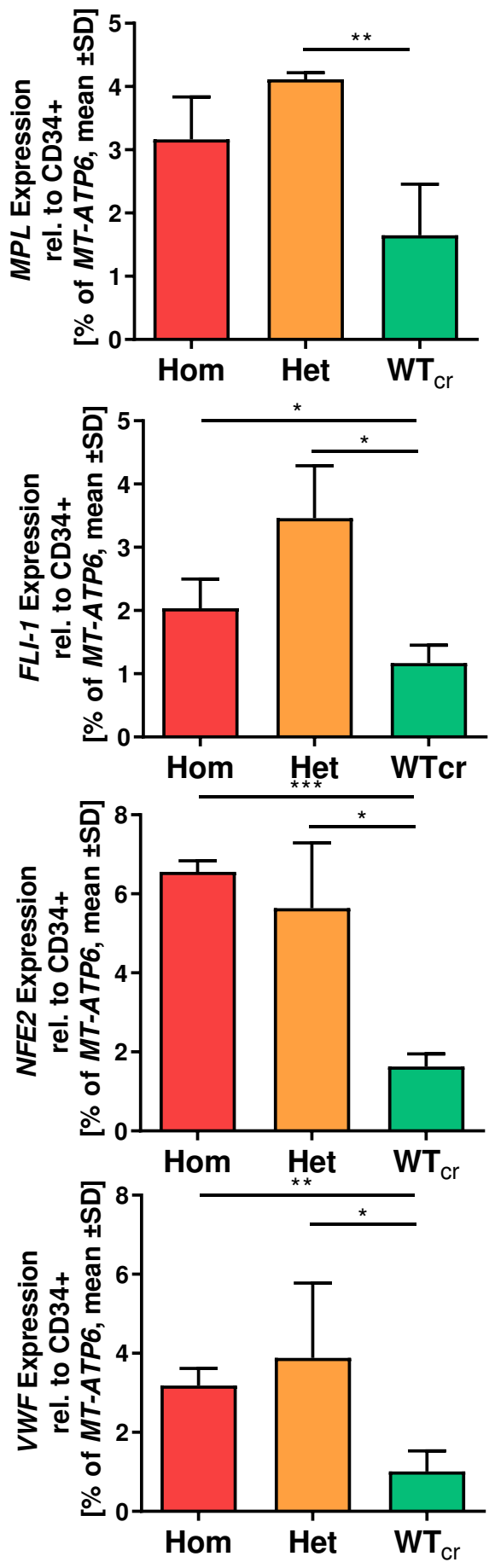
Figure 6
$A$
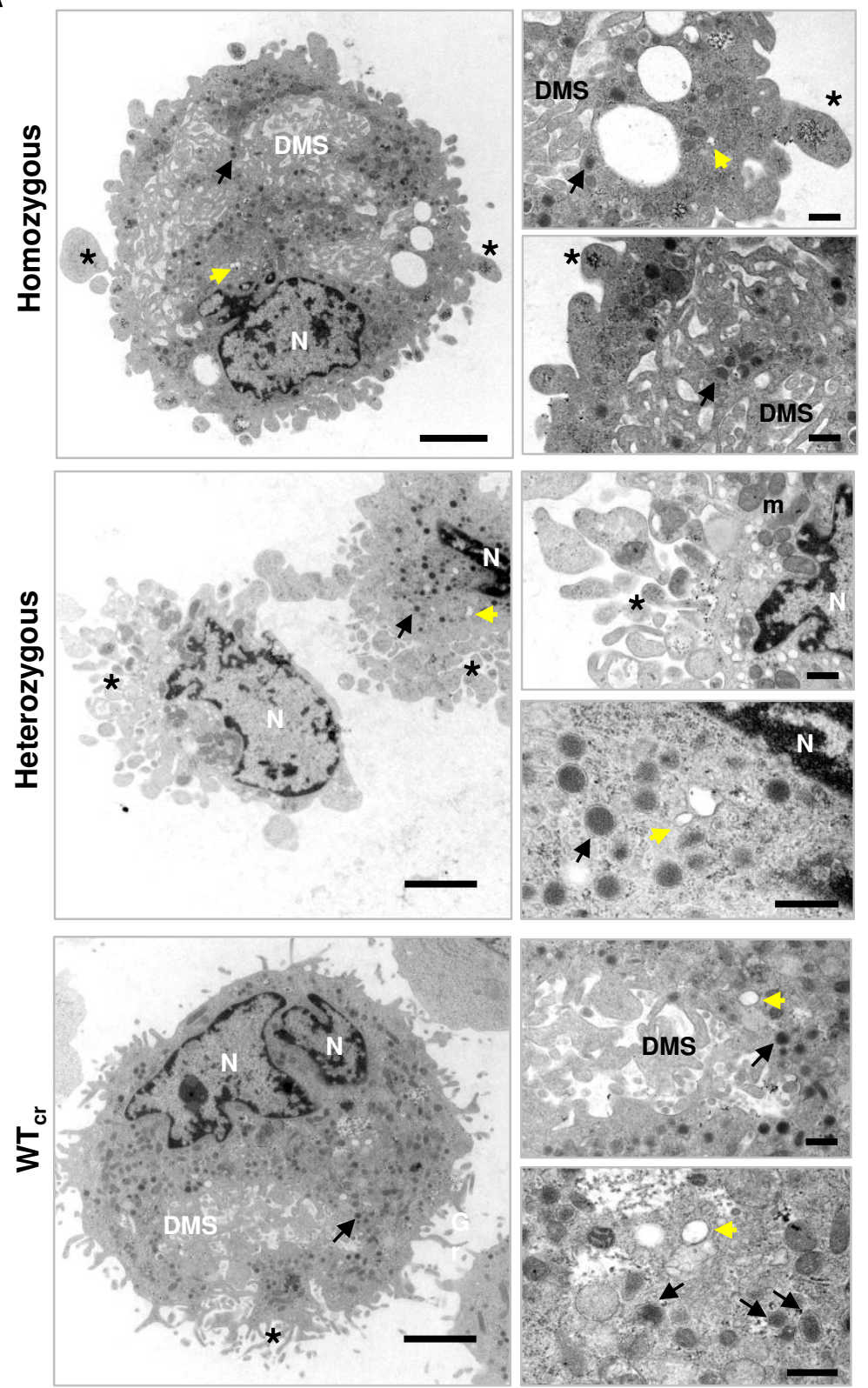

B
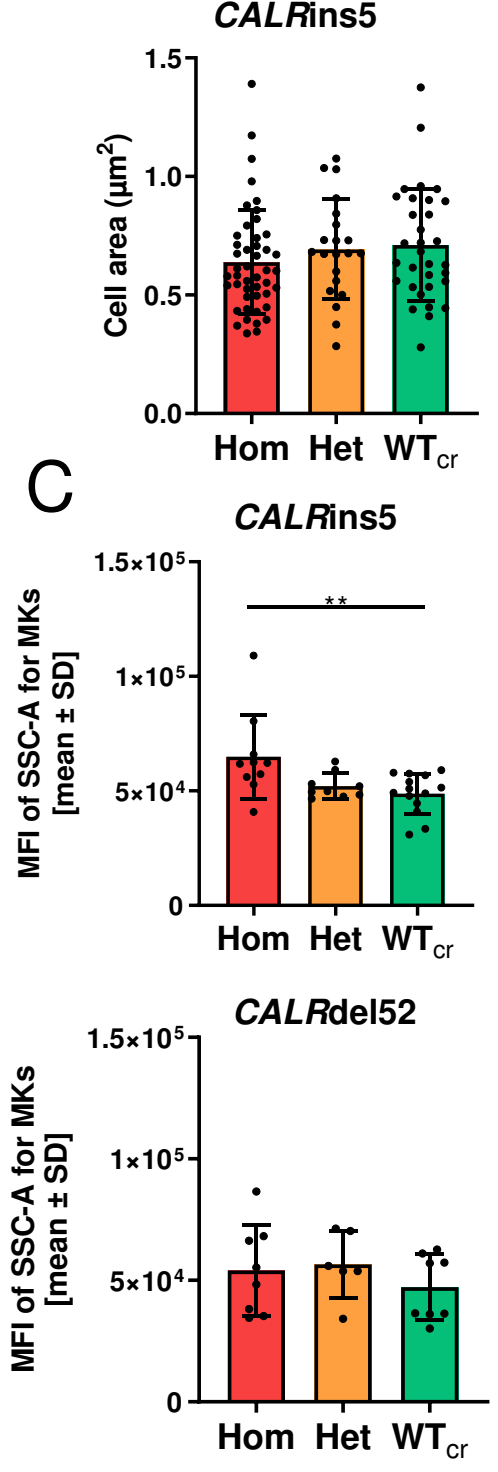

$D$
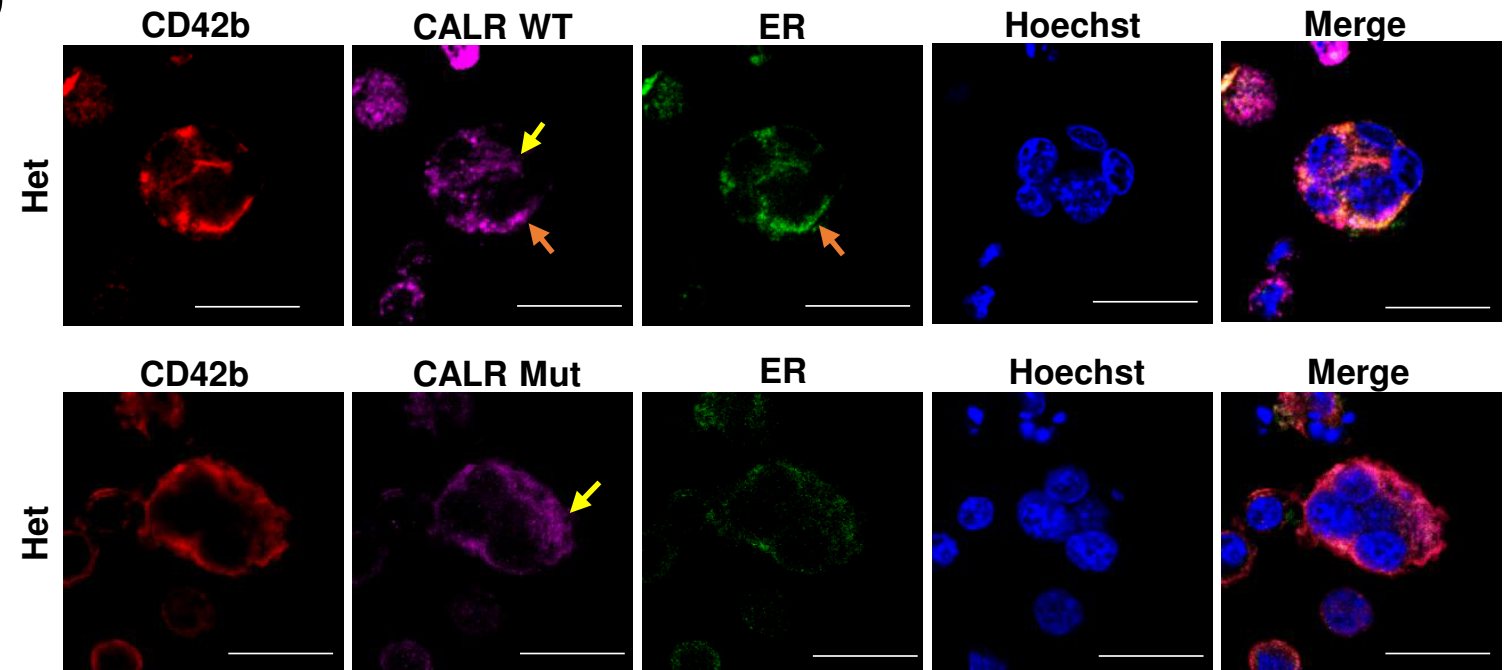

CALR Mut
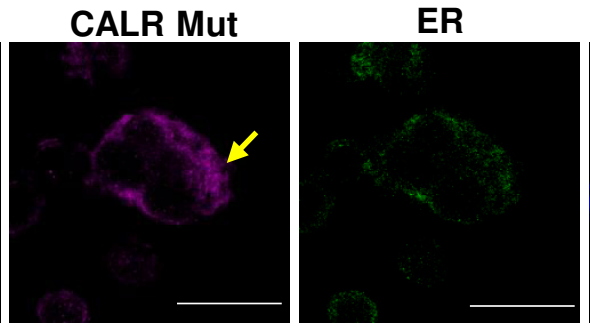

Hoechst

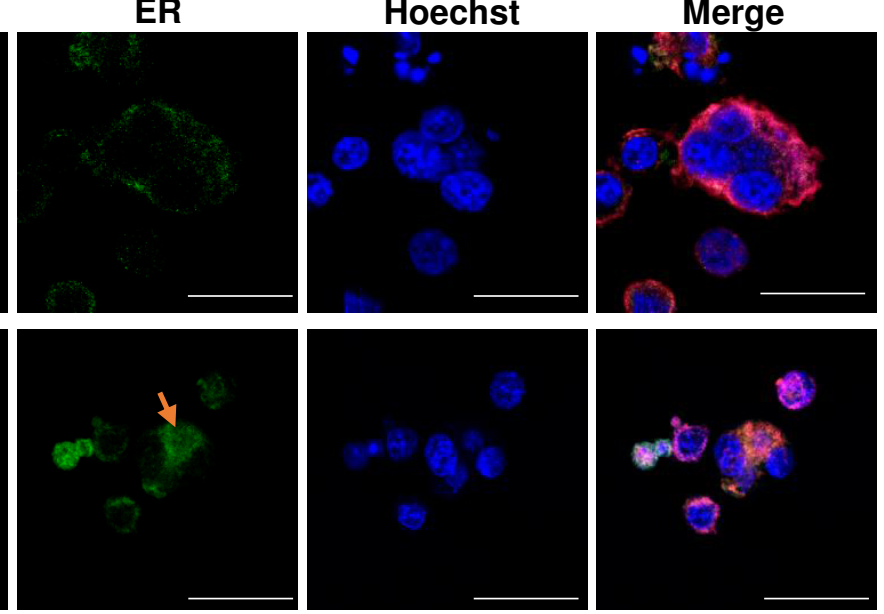

$\stackrel{\Xi}{\underline{I}}$
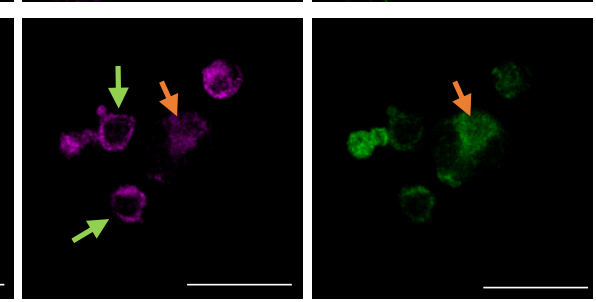
A

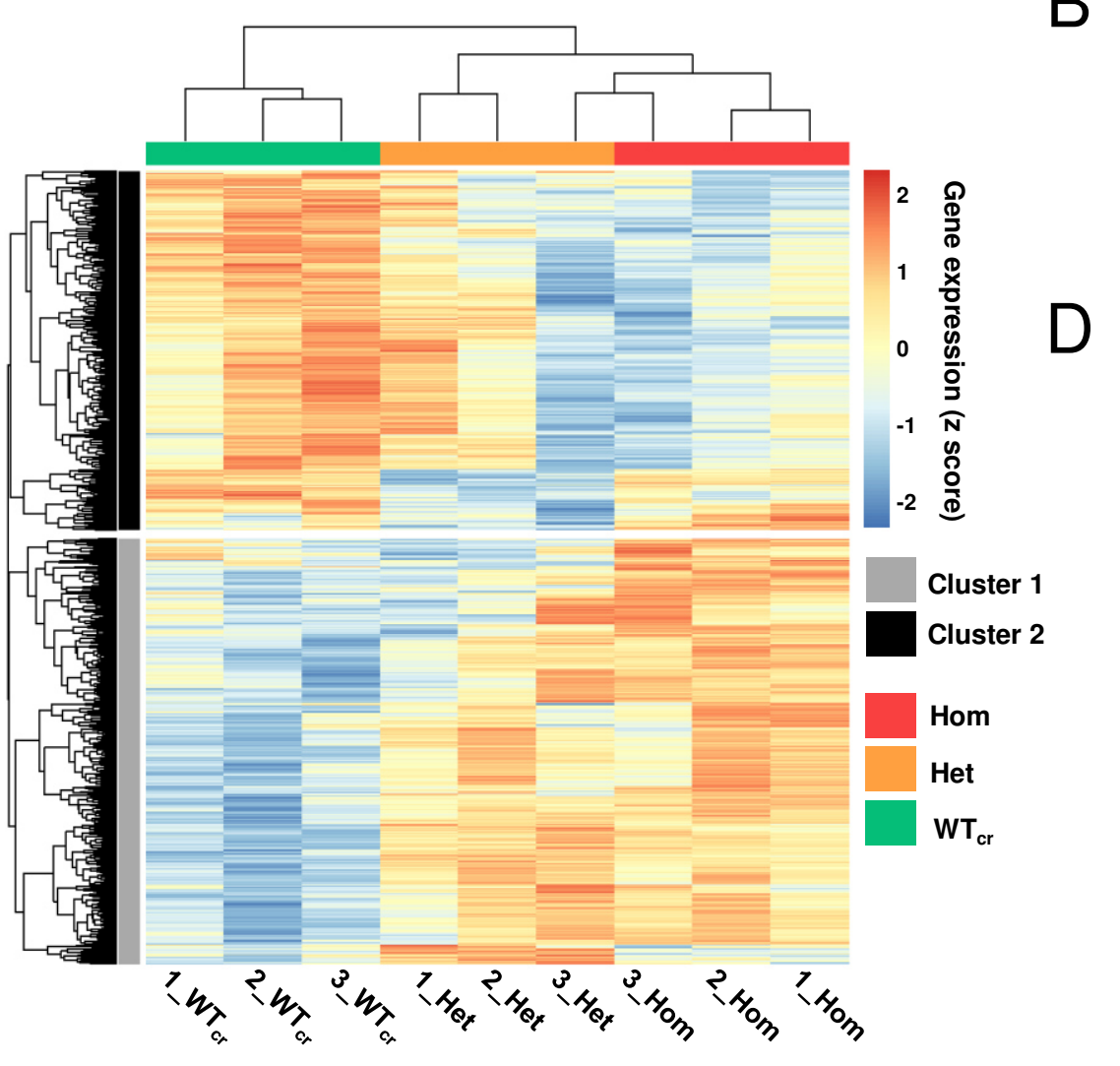

C

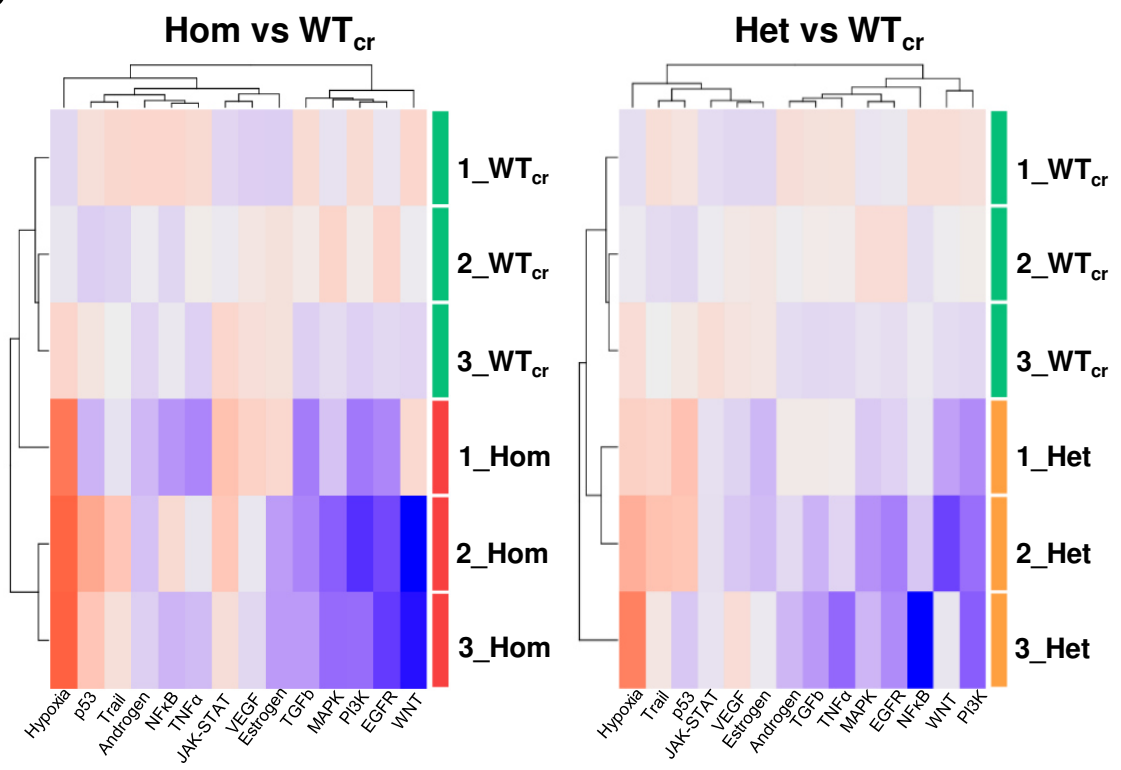

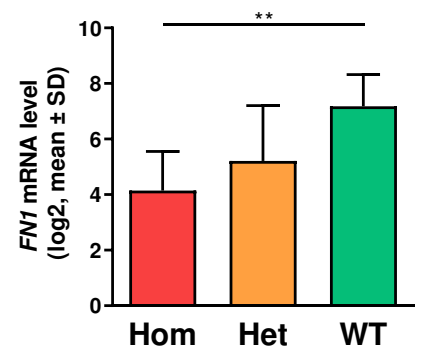
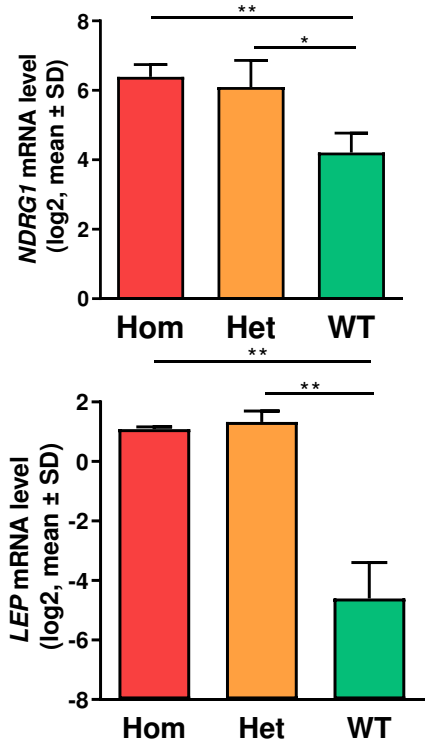

Activity

$=10$

Hom vs Het

0

$-10$

1_Hom

2_Hom

3_Hom

1_Het

2_Het

3_Het

\begin{tabular}{|c|c|c|c|c|c|c|c|c|c|c|c|c|c|c|}
\hline Comparison Pathw ay & Androgen & EGFR & Estrogen & Hypoxia & JAK-STAT & MAPK & NFkB & p53 & PI3K & TGFb & TNFa & Trail & VEGF & WNT \\
\hline Hom vs WTcr & 0,154 & 0,021 & 0,435 & 0,012 & 0,194 & 0,114 & 0,435 & 0,662 & 0,021 & 0,021 & 0,277 & 0,561 & 0,951 & 0,277 \\
\hline Het vs $\mathrm{WTcr}_{\mathrm{T}}$ & 0,380 & 0,177 & 0,300 & 0,177 & 0,380 & 0,177 & 0,380 & 0,551 & 0,032 & 0,330 & 0,380 & 0,300 & 0,551 & 0,300 \\
\hline Hom vs Het & 0,932 & 0,898 & 0,932 & 0,898 & 0,067 & 0,932 & 0,898 & 0,932 & 0,898 & 0,898 & 0,932 & 0,898 & 0,932 & 0,932 \\
\hline
\end{tabular}

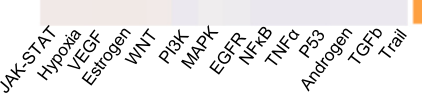

